\title{
EMI-aware Central Processing Point Deployment in Wireless Networks for e-Healthcare Services
}

\author{
by \\ Nick Majedi \\ B.Sc. in Computer Engineering \\ University of Isfahan, Isfahan, Iran, 2011
}

\author{
A Thesis \\ Presented to the School of Graduate Studies at \\ Ryerson University \\ in partial fulfilment of the \\ requirements for the degree of \\ Master of Applied Science \\ in the program of Computer Networks
}

Toronto, Ontario, Canada, January 2014

(C)Nick Majedi 2014 


\section{Author's Declaration}

I hereby declare that I am the sole author of this thesis.

I authorize Ryerson University to lend this thesis to other institutions or individuals for the purpose of scholarly research.

Author's Signature:

I further authorize Ryerson University to reproduce this thesis by photocopying or other means, in total or in part, at the request of other institutions or individuals for the purpose of scholarly research.

Author's Signature: 


\title{
Abstract \\ EMI-aware Central Processing Point Deployment for Wireless Networks in Hospitals
}

(C)Nick Majedi, 2014

\author{
Master of Applied Science \\ Computer Networks \\ Ryerson University
}

In this thesis, we introduce an approach to jointly minimizing energy consumption of wireless transmitters and the harmful interference on sensitive medical devices, and optimizing the central controller placement for collecting data from transmitters. More specifically, a multi-objective non-convex mixed-integer non-linear programming (MINLP) problem is defined and formulated to optimize transmit power of wireless users and CPP deployment along with guaranteeing minimized EMI to comply with the immunity level of medical devices. To solve this problem, an important step is to convexify it. Then we propose the use of a standard branch-and-bound algorithm. Also the program is coded in MATLAB with the help of OPTItoolbox to find the optimal solution. To conduct a numerical result analysis, we considered different cases within a single floor of a hospital and a few scenarios for a two-storey hospital building. The values of different variables are changed throughout the simulation and the results are compared. These variables include immunity level of sensitive medical devices, maximum transmission power of wireless users, floor attenuation factor, etc. Our results show the improvement in throughput and guaranteed immunity for medical devices, when the proposed formulation is deployed along with a branch-and-bound algorithm. 


\section{Acknowledgement}

I would like to express my sincere gratitude to my supervisor, Dr. Alagan Anpalagan for his continuous encouragement and support throughout my research. He was always motivating and generous with his invaluable time. It was a great privilege to work with him.

I would also like to acknowledge the Computer Networks department and the School of Graduate Studies at Ryerson University for their support in terms of financial aid, and work experience as a teaching and graduate assistant.

Thanks are also to my colleagues in the WINCORE lab and RRM+RAN Research Group. I am honored to be a part of this group where a team spirit truly prevails. I would especially like to thank Dr. Shaharyar Khwaja for his great help.

My special thanks go to my parents for their great support and encouragement during my Master's studies, and to my sister, Nikoo, who is always a great source of love and motivation. 


\section{Contents}

1 Introduction $\quad 1$

1.1 Motivation and contribution . . . . . . . . . . . . . . . 3

1.2 Thesis organization . . . . . . . . . . . . . . . . 4

2 Background $\quad 5$

2.1 Networks at hospitals . . . . . . . . . . . . . . . . 6

2.2 Cognitive radio-based communication networks f . . . . . . . . . . . . 7

2.3 Optimization problems and nonlinear programming . . . . . . . . . 8

2.4 Chapter summary . . . . . . . . . . . . . . . . . 10

3 Literature Review $\quad 11$

3.1 e-Healthcare services . . . . . . . . . . . . . . . . . 13

3.2 Technologies and standards . . . . . . . . . . . . . . . . . 19

3.3 Potential challenges in design and implementation . . . . . . . . . . . . . 21

3.4 Chapter summary . . . . . . . . . . . . . . . . . . . 26

4 System Model and Assumptions 28

4.1 Hospital environment . . . . . . . . . . . . . . . . . . 28

4.2 Transceiver model . . . . . . . . . . . . . . . . . . . . . . . . . . 33

4.3 Channel model . . . . . . . . . . . . . . . . . . . . . 35

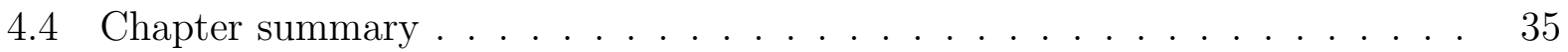


5 Problem Formulation $\quad 37$

5.1 Initial formulation . . . . . . . . . . . . . . . . . . 37

5.2 Reformulation ........................ . . . 42

5.3 Branch-and-bound algorithm . . . . . . . . . . . . . . 46

5.4 Chapter summary . . . . . . . . . . . . . . . . . . 49

6 Experimental Evaluation and Simulation Results 51

6.1 Simulation setup . . . . . . . . . . . . . . . . . . 51

6.2 Numerical results . . . . . . . . . . . . . . . . . . . 53

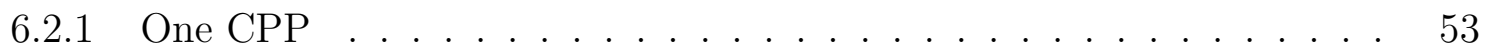

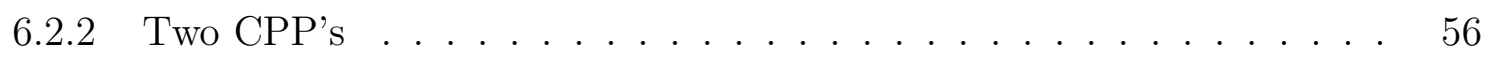

6.2.3 More Random SA's . . . . . . . . . . . . . . . . . . 60

6.3 EMI sensitivity . . . . . . . . . . . . . . . . . . . . . 61

6.4 Chapter summary . . . . . . . . . . . . . . . . . . . . . 64

7 Conclusion and Future Work $\quad 65$

7.1 Summary and conclusion . . . . . . . . . . . . . . . . 65

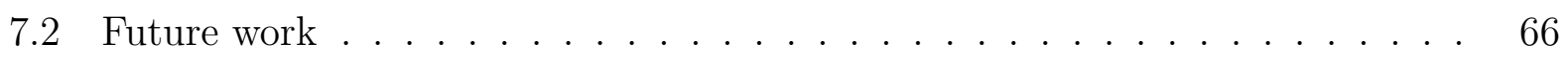




\section{List of Figures}

3.1 Time line of researches in e-health services . . . . . . . . . . . . . 14

3.2 Some of the elements of e-Healthcare. . . . . . . . . . . . . . . . . . 15

3.3 Services of e-Healthcare. . . . . . . . . . . . . . . . . . 16

3.4 QoS requirements for e-Health services . . . . . . . . . . . . . . . . . 24

4.1 CPP placement in multi-storey buildings. . . . . . . . . . . . . . . . . . 29

4.2 Horizontal and vertical views of an SA and hospital building, respectively . . 34

5.1 Flowchart of simulation process in MATLAB using OPTIToolbox. . . . . . . 48

6.1 Transmission power required by the user of and SA, located the furthest from its associated CPP for different number of SA's. This power satisfies the QoS requirement and EMI avoidance in the protected area. . . . . . . . . . 53

6.2 A 3D model of when a CPP is assigned to $2 \mathrm{SA}$ 's within the same floor. . . . 54

6.3 Effect of height of the floor on maximum transmission power required by the user of an SA, located at the furthest point from its associated CPP, for different number of SA's. . . . . . . . . . . . . . . . . . 55

6.4 CPP Placement for 2 SA's and 2 CPP's and the distance of the worst user. . $\quad 57$

6.5 CPP coverage and output power level difference for two different cases. . . . 58

6.6 CPP positioning and coverage for $3 \mathrm{SA}^{\prime}$ s on a single floor and $2 \mathrm{CPP}$ 's. . . . 59

6.7 CPP positioning and coverage for 4 SA's on a single floor and 2 CPP's. . . . 60 
6.8 Comparison between the average transmission power of SA's in the presence of 2 CPP's (the topology shown in Figure 6.7) and 1 CPP. . . . . . . . . . 61

6.9 Semi-random SA orientation for two SA's and one CPP . . . . . . . . . . . 62

6.10 Semi-random SA orientation for three SA's and one CPP . . . . . . . . 63 


\section{List of Tables}

4.1 Notations . . . . . . . . . . . . . . . . . . . . . . . 31

6.1 Numerical results for when there is one CPP for different number of SA's . . 55 


\section{Chapter 1}

\section{Introduction}

The improvements in computing and communication systems have had great influence in different aspect of our lives. One of their most important impacts was on healthcare. RFID, wireless sensor networks and information management frameworks are among the technology platforms used in healthcare applications. In this context, sensors, electrical devices, networks and wireless communication systems can replace traditional systems in healthcare environments, as trustworthiness of these technologies have reached to an acceptable extent. This will minimize both operational and capital costs by increasing efficiency while improving the delivery of service to patients [1]. As an example, conventional body temperature sensing can be replaced by small temperature sensors with integrated wireless transceivers for more precision and easy-to-deploy structure.

Utilizing hospital information systems (HIS), radiology information systems (RIS), picture archiving, communication systems, etc within hospitals and clinics has proved to increase the efficiency of the caregivers. The objective of this is to make their work easier in terms of, for instance accessing patient's medical records more quickly for treatment [2]. Therefore, the term e-Healthcare is introduced. Regardless of all the tremendous advances in this field, there still remain unexplored aspects of it, especially considering the challenges in communication and networking. As an example, processing large amounts of real-time 
data gathered from sensors and transferring them to a central station requires development of a middleware that can handle delivery of critical data on-time. Security is another issue in e-Healthcare. It involves confidentiality, integrity and availability of data and systems.

Devices within hospitals can use multiple access methods for accessing the channel and transmitting their data to the central station. Prioritization and on-demand access to the channel for devices with realtime data transfer is usually a necessity. Regardless, lower priority devices should not confront bandwidth starvation.

The energy efficiency of battery powered wireless devices has been explored for different network strategies. As a good example in [3], the authors investigate techniques to optimize the number of base stations and their locations in order to minimize the energy consumption.

Of other important challenges within hospitals is the harmful impact of wireless transmissions on sensitive medical devices. This can be addressed by controlling the maximum transmission power of wireless devices and keeping the more sensitive devices as far as possible from the transmitters. In this sense, minimizing the transmission power reduces the harmful interferences and increases the life time of battery powered nodes. However, this power minimization might decrease the throughput of wireless transmitters. Quality of service methods can be deployed to maintain desired throughput for such nodes.

Another commonly used terminology for integration of information and communications technology within healthcare, is m-Health or mobile health. Patient monitoring is made easier as they do not need to stay in hospitals or interrupt their daily life just for being monitored. Wearable monitoring devices give the patients the freedom to continue their normal life while being monitored and the necessary data to be transferred to hospital for required diagnosis [4].

In this thesis, we will initially provide a review of e-Health and m-Health services, their commonly-used and emerging technologies, and challenges in implementing such services. The main part of our work, however, is addressing some of the challenges mentioned in the discussion above. More particularly, we will focus on jointly minimizing energy consump- 
tion of wireless transmitters and the harmful interference on sensitive medical devices, and optimizing the central controller placement for collecting data from transmitters.

\subsection{Motivation and contribution}

This work is motivated by the works proposed in [5] and [6]. The former investigates the optimal deployment of femtocell base stations in commercial buildings. The model considers optimizing the placement of femtocell base stations as well as power control to extend the battery life of wireless transmitters. Also the various constraints in base station installation are taken into account. Based on this model, in this thesis, the problem is formulated as a mixed-integer nonconvex programming problem. It is then transformed into an equivalent mixed-integer convex programming problem to facilitate design of an optimization algorithm. The algorithm used to obtain a solution is based on a branch and bound framework and convex programming relaxations $(\mathrm{BB} / \mathrm{CPR})$.

In [6] the authors discuss the power control and electromagnetic interference avoidance in hospital environments. It defines three types of users according to the importance of their transmitted data and wireless transmission capability. The main focus of that paper is on transmission scheduling and shaping the power consumption of the so called secondary users in STDMA networks. Their objective is defined as maximizing the spectrum utilization and minimizing the power consumption of secondary users considering electromagnetic interference constraints for medical devices. For this purpose, an enhanced greedy algorithm approach was proposed. However, optimized deployment of central controllers in hospital buildings, taking EMI minimization into account, is under-explored. Therefore, in this study we direct our research towards mathematical modeling of a joint central controller placement, uplink power minimization of users, and EMI minimization for EMI-sensitive devices within hospitals. Our proposed central processing point (CPP) placement planning is challenging in real world due to the building sizes, different layout of rooms, structure and room separators 
(walls/floors).

The key contributions of the work in this thesis are:

- Optimizing central controller deployment and assignment of CPP's to service areas $(\mathrm{SA}$ 's)

- Minimizing transmission power of wireless users

- Minimizing the EMI to sensitive medical devices

- Formulating a mixed-integer non-linear problem to address all the point above

- Coding in MATLAB using OPTI-Toolbox which integrates an improved branch and bound algorithm

- Analyzing the impact of various factors within different scenarios

\subsection{Thesis organization}

In this thesis, we first give an overview of networks used in hospital environments and the intelligent wireless transmission systems that are capable of handling highly sensitive, realtime data. Secondly, we will have a review of integration of telecommunication in eHealthcare with a focus on the services, technologies, applications and challenges in their implementation. Next, we introduce our system model, describing the assumed hospital environment, the transceiver model and the channel model in such environment. After discussing the modeling and assumptions, the formulation of our problem is explained in details along with an overview of the branch and bound algorithm, and the toolbox in MATLAB used to solve the mixed integer non-linear programming (MINLP) problem. We further provide numerical analysis of results obtained from the simulation. At the end comes the conclusion of this thesis and the future work proposed. 


\section{Chapter 2}

\section{Background}

The optimal deployment of central processing points (CPP's), also referred to as base stations, in field hospitals are of great importance. Wireless devices within hospitals can cause harmful electromagnetic interference to some devices in hospitals. This interference can cause faulty readings of those devices, malfunctioning, or even restarting and shutting down. Optimal CPP deployment ensures network coverage and improves power consumption of users so that their lifetime is increased. The application of CPP deployment is not limited to field hospitals and can be implemented in large hospital buildings of multi-floors, clinics, and other health centers.

We assume a hospital building to have multiple service areas (SA's) where medical devices

are operated. Inside each service area, there exists a protected area which is sensitive to electromagnetic interference (EMI). In other words, EMI sensitive medical devices operate in this area. Electronic components of these devices show performance deterioration when exposed to electromagnetic field. The effects include automatic shutdown, automatic restart [6], altered monitored results, etc. Examples of these devices are defibrillators, incubators (as life-supporting devices), infusion pumps and ECG monitors (as nonlife-supporting devices) [6]. Users are considered to be devices with wireless transmission, for example telemetry devices. Central processing points are nodes controlling the wireless transmission of users 
and collecting data from them.

CPP placement optimization in hospitals is important because it has a direct impact on the power consumption of the users. In other words, we can optimize uplink power of users in hospitals. In our problem, we aim to determine the coordinates of CPPs (i.e., x, y and z) and meet the quality of service (QoS) requirement of each user. This model considers floor attenuation factor (FAF), CPP installation restrictions and translates to minimizing the worst user's transmission power (a min-max problem).

\subsection{Networks at hospitals}

The healthcare monitoring provided by hospitals to the patients during their stay at care units, is a sensitive procedure since an emergency management requires rapid access to facilities or personnel capable of delivering treatment to the patient in severe conditions. Hospitals, usually do the initial care, assessment, and monitoring in emergency rooms until the patient reaches a stable health condition. Then the patient will be transferred to the care unit. The care units of today's hospitals differ from those of old ones. For example, consider a patient who is transferred to a care unit and has just passed a heart stroke. In the past, voluntary acts of such a patient were required. This voluntary act could be sounding a bell or pushing a button. Whereas in nowadays' hospitals, advanced monitoring systems are introduced which can increase the patient's autonomy and safety, and also granting mobility to patients, staff and relatives of patients [7].

A common solution of autonomous wireless monitoring is based on using radio frequency (RF) networks. Although these networks can provide automation to patient monitoring as pros, they have some cons as well. These cons include electromagnetic disturbance and security. Optical wireless or infrared (IR) technology can solve some of the downsides of RF technology. This technology has many advantages such as being license free, compact, low cost, and demands low power consumption with high security. On the other hand, its 
disadvantages make this technology less desirable. IR technologies have been studied for indoor environments and local area network applications for many years. The main setup requires point-to-point transceivers. Line-of-sight (LOS) propagation is the base of point-topoint systems and requires an accurate alignment between optical transmitter and receiver. This is not desirable in indoor environments [7].

The development of wireless communication systems has caused a huge change in the development of small-size, low-power sensors in recent years. Deploying computer networking in hospitals is one the main goals of today's health service developers. As an example, medical body area networks (MBAN's) are interconnected wireless sensors which collect and transport vital signs and medical information of users (patients). The MBAN transmitters are carried by patients to different locations of a hospital, from patient rooms to corridors and even to rest rooms. Movements of patients change the propagation characteristics of the resulting channel which makes a complicated wireless network system and introduces some limitations to the use of these networks. The more the characteristics of channels are known, the more understanding of performance limits is achieved [8]. The short-range private networks are suitable for public places such as hospitals. The indoor short-range wireless communication with built-in high-speed radio access has led to the wireless LAN extensions. The common range of operation for WLAN in indoor applications including hospital environments, is at $2.4 \mathrm{GHz}$ and $5.8 \mathrm{GHz}[9]$.

\subsection{Cognitive radio-based communication networks}

Cognitive radio is used for transceiver systems that are able to alter some parameters when in operation, in order to optimize their context-based operation [10-12]. This is accomplished using methods such as spectrum sensing. An intelligent artificial mechanism called "cognitive engine" works as a means that senses spectrum, makes decision and adapts the transceiver's behavior [10]. 
Cognitive radio networks are the outcome of recent advancements in intelligent, adaptive wireless device communications. In the context of cognitive wireless networks, different users are defined. Often, users are classified as primary users (license-holders of a spectrum band) and secondary users (cognitive radio users). The cognitive users communicate by using their cognitive capabilities in order not to interrupt the primary users. There are still a lot of studies conducted involving cognitive radio networks as it is an emerging area of research. The idea originally evolved from the lack of spectrum under the existing spectrum management policies [13].

The federal communications commission (FCC), controls the licensing of the spectrums in the US. Although most of the frequency spectrum useful for wireless communications have already been licensed by FCC, still there a few unlicensed bands such as industrial scientific and medical (ISM) band over which the common Wi-Fi devices transmit. The lack of frequency spectrum has led the world to a spectral crisis, and this is getting more serious as time passes. The license holders have an exclusive right to transmit over their spectral bands. On the other hand the unlicensed bands are limited in number and are filling up fast. Here glows the intelligence of cognitive radios as they can sense the presence of the primary users and adapt their transmission to make sure that the performance quality of primary user is maintained.

\subsection{Optimization problems and nonlinear programming}

Optimization is present in every aspect of today's life and has become an inevitable part of it. It sometimes appears as minimization, as in minimizing the mistakes when playing a piece of music on an instrument, and sometimes as maximization, e.g., maximizing the efficiency and performance in an engineering project. Thus the applications of optimization span across both science and practice. Regardless, whatever the case is, it can be formulated in a generic form [14]. Many of these applications in science and engineering utilize mathematical 
modeling. A mathematical model consists of a set of mathematical relationships which represent an abstract model of the real world system. These relationships include equalities, inequalities and logical conditions [15].

After modeling a problem, in order to find an optimal value of interest, an optimization algorithm must be used. There are a number of optimization algorithms, each suitable for particular types of optimization problems. In many cases, the evaluation of the objective function is extremely resource consuming in terms of computational power and time. Therefore, the more efficient the algorithm in use, the more time and money are saved. Efficiency of algorithms is measured by their computational complexity [14]. Optimization algorithms are normally iterative. They start with an initial guess of the decision variable and generate improved estimations. When they terminate, the results will hopefully be considered as a solution to the problem. After applying an optimization algorithm to a model, it has to be verified whether the algorithm has succeeded in finding a solution. In many cases, there exist optimality conditions for checking to see if the current results are the solution of the problem [16].

Our focus in supporting this thesis work will be on a nonlinear and mixed integer optimization model which obviously requires taking advantage of mixed-integer nonlinear programming (MINLP) techniques and algorithms. A lot of optimization problems in real world are nonlinear. If there exist integer variables as well as nonlinear terms in the objective function and constraints, we are dealing with MINLP. Binary variables can be used to represent assignment, existence, occurrences, etc. Discrete variables can model dimensions and number of occurrences, where continuous variables would be used to show inputs and outputs to the system, as an example [15]. 


\subsection{Chapter summary}

In this chapter, we briefly introduced the environment considered in this thesis work, with a touch on the importance of deployment planning and interference avoidance in wireless sensor networks. Then, we described the contemporary wireless and computer networks within hospital environments. Another important topic that was discussed in this chapter, is the cognitive radios and the networks that benefit from this technology. Finally, the optimization problems are introduced with intuitive real-life examples. 


\section{Chapter 3}

\section{Literature Review}

The integration of digital data processing, computing and communication technology into the traditional healthcare services is known as e-Healthcare [17]. With the continuous growth of elderly population, there have been a rise in need for more cost effective and reliable healthcare services. These services can provide both patients and caretakers with uninterrupted monitoring, appliance control, medical data access, memory enhancement, emergency communications, etc. The terms used in this concept include e-Health, tele-Health and mHealth. While e-Health and tele-Health are usually used interchangeably, they have evolved from the term "tele-medicine". At its preliminary phase, tele-medicine made possible the transmission of electrocardiograms over phone lines other than enabling individuals to call doctors. With the evolution of digital techniques, communications and information processing, a lot of burdens are removed in this context. Moreover with the rapid development of the Internet since late 1990's, various health applications became available remotely and hence the term e-Health was introduced [18]. With the further improvement of mobile com-

munication technologies and emerging handheld devices, the term m-Health was coined. It refers to the integration of mobile telecommunications and multimedia devices with health services [19]. m-Health is sometimes considered as a sub-segment of e-Health. Although there is no agreed-upon official definition, usually the devices used in m-Health are mobile 
phones, PDAs and tablets whereas e-Health only makes use of computers and communications systems [20]. In the past decade, the main focus of research community has been on integrating wireless communication technologies, artificial intelligence and sensors into medical devices [21].

With the advancement of medical devices and integration of communication and computing systems with such devices, the whole world can be considered as an interconnected village. For example, Figure 3.2 summarizes some of the services of e-Healthcare and illustrates the integrity of these services within a typical hospital, home and tele-health environment. Considering hospital as the center of health services, within a hospital there are several doctors, caregivers, diagnostic and monitoring devices. The data from these devices or from reports of the doctors/caregivers are stored in a local database, and synchronized with a central electronic health records (eHR) database. Patients have various sensors attached to them. Some of these sensors acquire vital data and have to transmit in real-time. Other than patients, rooms and hospital beds have different sensors, e.g., proximity sensors, occupancy sensors, pressure detectors, etc. These sensors and transmitters constantly send their accumulated data to the controllers which are connected to the central monitoring room. With the improvement of wireless transmission systems, patient monitoring and tele-health services are now possible in remote locations. Authors in [22] discuss some of the benefits of these services including lowering the response time of caregivers in emergencies, improving the life quality of elderly, enabling the large-scale filed studies of human behavior, and early detections as a result of real-time monitoring of patients.

Sometimes there is need to provide emergency services in large disaster areas [22]. The tsunami at pacific coast of Japan in 2011 and the earthquake at the city Tabriz of Iran in 2012 are examples of mass-casualty disasters. In such cases, a field hospital can be set up in the locations that have a large number of injured people. Infrastructure-less wireless nodes and sensors are then used to acquire help from remote doctors and access patients' eHR. Adding up to the benefits of e-Healthcare, we refer to a project called Whole 
System Demonstrator Programme, funded by the Department of Health in England [23]. The purpose of the project was to find out how technology can help people improve their health while maintaining independence. The results have proved that if delivered properly, tele-health can substantially be beneficial in promoting people's long-term health, improving the patients' lives, etc [23]. These results include [24]:

- $45 \%$ reduction in mortality rates

- $21 \%$ reduction in emergency admissions per head

- $14 \%$ reduction in elective admissions per head

- $15 \%$ reduction in emergency department visits

- $14 \%$ reduction in bed days per head

- $8 \%$ reduction in tariff costs per head

The timeline diagram in Figure 3.1 illustrates the scope of research done in e-Health in recent years. The fact that monitoring applications of all kinds comprises most of the work is very fortunate and entails important advances in pervasive, in-home, and in-hospital monitoring. This has had remarkable impact on life of elderly and made possible the smart healthcare to chronically ill and children.

\section{$3.1 \quad$ e-Healthcare services}

There is a wide variety of services offered under e-Healthcare framework. Figure 3.3 summarizes these services. As can be noted from the summarized list, there have been lots of research activities over the last decade in computerized e-Health service delivery mechanisms with the use of information and communications technology (ICT). In the following we discuss them. 


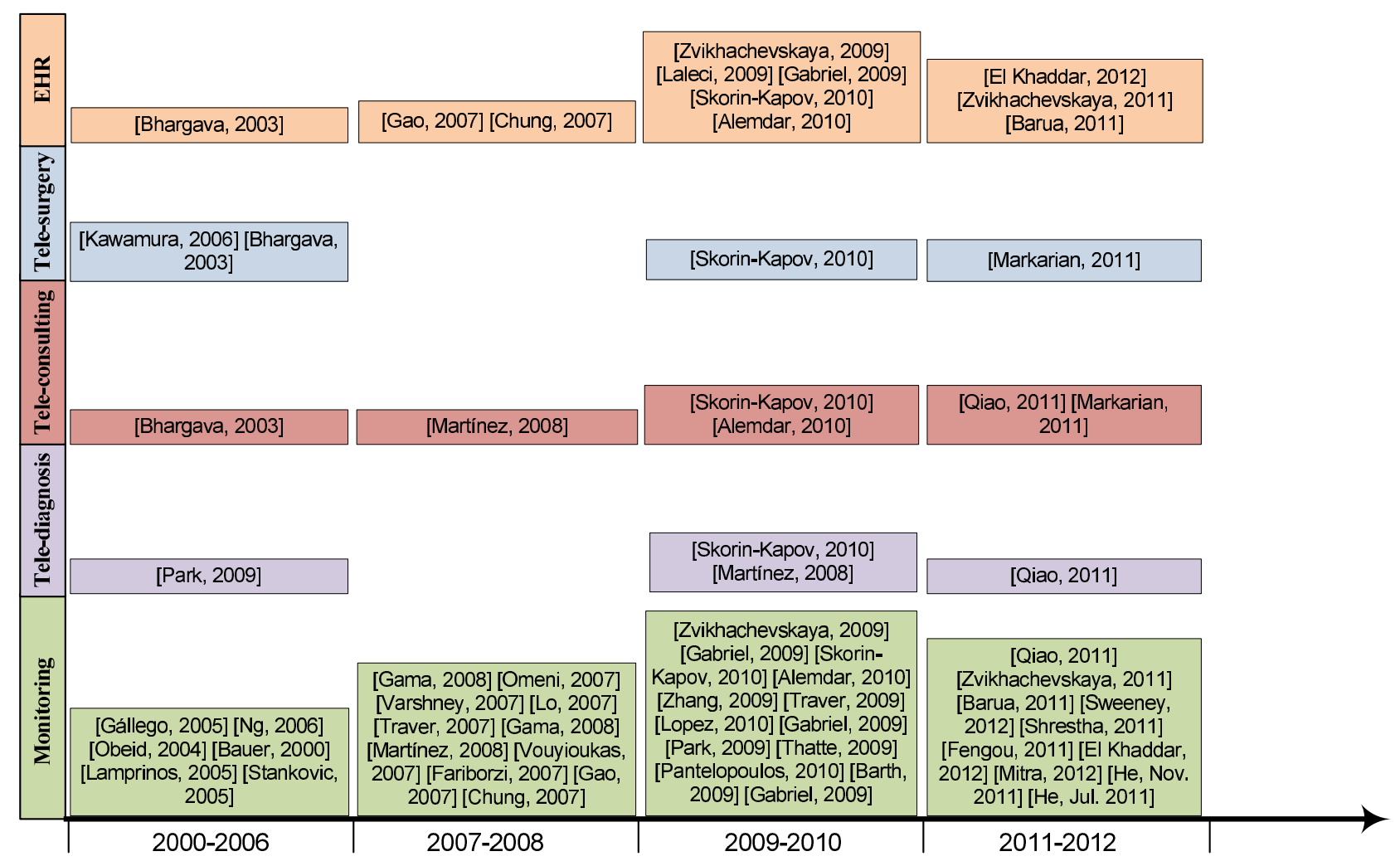

Figure 3.1: Time line of researches in e-health services

Monitoring patients consists of pre-hospital monitoring, in-hospital monitoring [25, 26], and pervasive health monitoring [27]. Pre-hospital monitoring usually implies observing patients on the way to hospital. This is done inside equipped ambulances and helicopters in some cases. Depending on the technology of use, these vehicles might have different capabilities including tools for electrocardiography, cardiac dysrhythmia detection, blood pressure monitoring, capnography and defibrillation pads [28]. In-hospital monitoring in eHealthcare comprises of vital signs monitoring, electrocardiography, capnography, child birth monitoring, blood pressure, glucose and flow monitoring, body temperature monitoring [29], etc. Some of the used devices for these purposes transmit realtime wireless data which should access the channel whenever they have data available, such as telemetry devices $[30,31]$. 
One of the important characteristics of e-Healthcare is that patient data must be available to caretakers in real-time without the constraints of time and location. This is referred to as pervasive health monitoring $[32,33]$. Pervasive health monitoring is a fast-growing topic in e-Healthcare which improves prevention and early detection of diseases. One of the biggest challenges within this context is quality of service (QoS) as data streams from various sensors have different priorities and an extra delay in high priority packets may incur fatal consequences [34].

In [32], authors investigate different applications, requirements and challenges of pervasive healthcare. Wireless infrastructures are evolving constantly, thus improving the quality

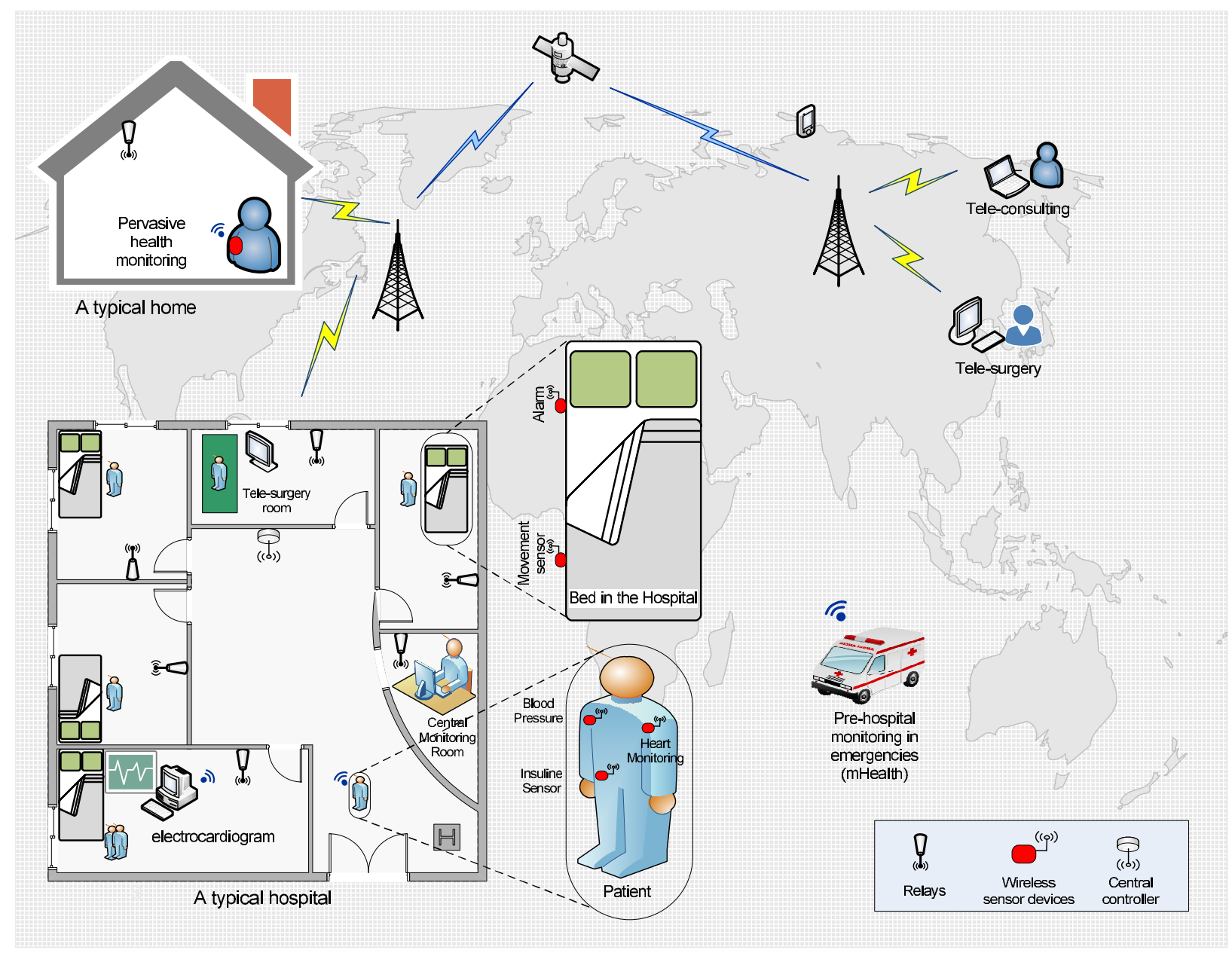

Figure 3.2: Some of the elements of e-Healthcare. 


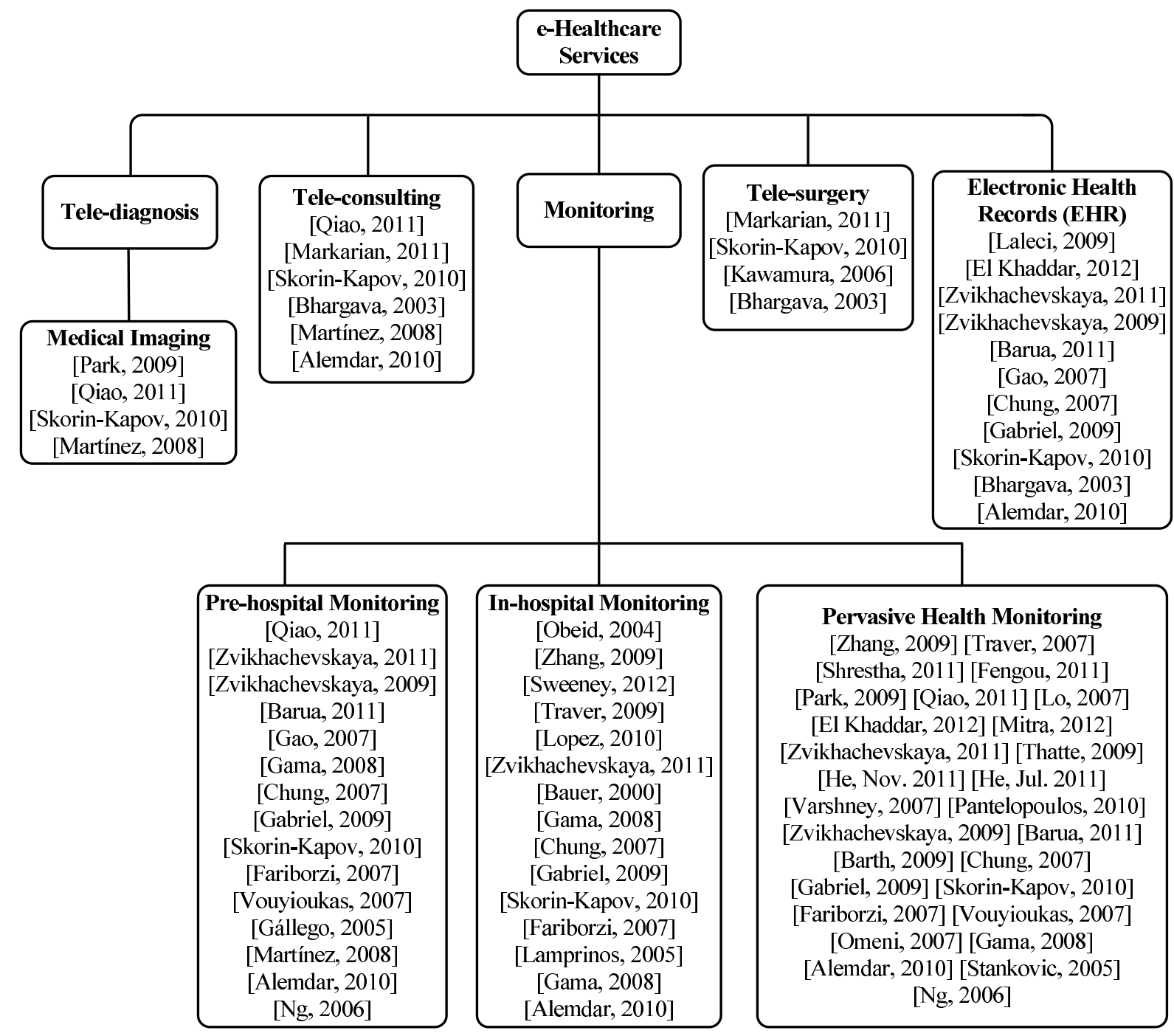

Figure 3.3: Services of e-Healthcare.

of transmission and coverage in existing and emerging healthcare applications, lowering the workload on caregivers and reducing the overall cost of health services. For instance, patients' medical information can be accessed anytime and anywhere using wireless networks and appropriate devices [35]. It has been reported in [32] that pervasive health monitoring eliminates the restraints of time and location and that patients would receive help much faster whenever they need it. Also context-aware intelligence in pervasive services would reduce false alarms.

One of the major applications of pervasive health monitoring is in-home monitoring 
[36]. The need for health services for elderly is rising as the population of world ages increasingly. Exploiting the capabilities of e-Healthcare would also help such patients to control the appliances, equipments and tools at home, to remember to take their drugs, to look up medical information and to communicate with caregivers when necessary and in emergencies. Meanwhile, the health status information of these patients can be updated in patient databases [21]. Other applications of in-home pervasive healthcare are fall/movement detection, location tracking and medication intake monitoring [21]. Integrating intelligent medical microsensors into wireless communication networks improves the life quality by providing complete observation of patients without affecting their normal life which causes lowering of the costs as well [37]. High speed wireless communications enable real-time monitoring of neural signals which can be used in implantable and wearable wireless sensors. This will be helpful for patients suffering from paralysis, blindness or deafness [38].

Wireless personal area networks (WPANs) such as ZigBee networks can be used to develop a home health monitoring platform in which the devices are mostly non-identical in terms of data rate and/or packet size $[39,40]$. In such a network, the IEEE 802.15.4 MAC can be used in beacon-enabled mode, which supports guaranteed time slot allocation for time-critical data transmissions [39]. Authors in [40] propose a new frame work architecture for low cost personalized e-Health services in which standardized services are deployed over next generation IP networks. At a smart home environment, tele-monitoring consists of continuous bio-sensing of patients, context-sensing of the living conditions, dynamic creation of the appropriate group of eligible subject (e.g. doctor, relative, nurse) to deal with the situation, and transmission of information or alarm signals to the eligible subjects [40]. In [41], tele-monitoring is described as transmission of a patient's vital bio-signals and other related data which are used in treating chronic diseases or post-hospital home care. This may involve multi-parametric signal transmission including ECG, blood pressure, saturation of peripheral oxygen, glucose level, patient's activity, air temperature, humidity and air pressure [41]. 
Authors of [42] have provided an overview of development of body sensor networks (BSN) which are proposed to improve patient care, manage chronic diseases, promote lifelong health for elderly and maintain a truly pervasive monitoring and sensing environment. In [33] authors present wearable wireless body area networks (WWBAN's) as being capable of providing instantaneous health status to their users through different sensors. An appealing topic in WWBAN is coupling of pervasive communications with lightweight portable devices to form pervasive health monitoring so that healthcare personnel can access patient data, review and update them seamlessly. The benefits due to the integration of ubiquitous computing in healthcare include mobility of healthcare, continuity of support in monitoring and treatment, patient status report through wide range of sensors in a home environment, improved patient satisfaction through self-management of the healthcare process, reducing medical errors, remote access provisioning to medical facilities and specialists, etc. [33].

Tele-diagnosis is a service which uses simple applications to facilitate point-to-point asynchronous communication [43-45]. This service is of high importance in areas that lack expert medical personnel. Usually the remote sites send requests to a medical center and transmit the required data regarding a diagnostic examination, e.g., medical images. The specialists review the data and send back a diagnostic report to the requesting parties. Teleconsultation, in contrary to tele-diagnosis, is generally defined as synchronous analysis and manipulation of medical data, which requires high QoS $[41,46]$. It involves an environment where remote parties (medical experts) can view the same set of medical data at the same time and exchange comments in real-time [43,47].

In tele-surgery, sometimes termed remote surgery [48], as the name implies, the surgeon is in a remote location and surgery is performed using a robotic system controlled by the surgeon. This service requires robotics in conjunction with a high speed communication system as streaming images need to be transferred in real time. The QoS requirements are very strict as the service is sensitive to delay and data loss [41]. Public telecommunication lines, such as Internet, can be used in connection with surgery environments to make tele-surgery 
a more commonly employed method, although current instances usually use a dedicated line in order to lower the delay and data loss [49]. Tele-surgery would lower the dissimilarities in medical treatments over different domains and also eliminates the risk of infection for doctors [50].

Electronic health record (EHR) is a systematic method of collecting and maintaining patients' data which makes it possible for the health records to be accessible from different hospitals or any other caretaking centers [51]. EHR can contain a wide range of data. Authors in [41] investigate the QoS requirements for EHR and introduce it as a delay tolerant e-Healthcare service. The delay depends on the amount of information to be transmitted but having a reliable transmission with no packet loss is a strict requirement $[52,53]$. EHR is accessed at anytime, either it is an emergency or not [41].

\subsection{Technologies and standards}

There is a wide variety of technologies and standards being used in the delivery of wireless e-Healthcare systems. We will discuss some of them in what follows.

3G is a large-scale wireless network and mobile computing solution, with this technology, healthcare personnel are able to load vital patients' information anywhere and anytime within the healthcare network. For instance, 3G technology is widely used for healthcare personnel to access the hospital database in an ambulance [54]. Wireless Local Area Networks(WLAN's) are being used very often in lots of situations today as well as for communications between medical devices [55]. WLAN's and cellular systems $(2.5 \mathrm{G}, 3 \mathrm{G}$ and beyond $3 \mathrm{G}$ ) are combined into a flexible and heterogeneous network, which makes m-health services more accurate, sufficient and reliable [33] for both fixed and mobile m-health users in a wireless metropolitan area network environment [49]. 


\section{Body sensor networks}

Body sensor network (BSN) is a term used to describe the application of wearable computing devices. This will enable wireless communication between several miniaturized body sensor units (BSU's) and a single body central unit (BCU) worn on the human body. With the combination of biosensors and wireless networks, medical personnel are able to pervasively monitor patients' health, behaviors, physiological and contextual parameters at all times [56]. Generally, in BSN's, low power radio is being used to minimize the radiation to human body and reliable and secure wireless links are also being used to transmit sensitive physiological data.

\section{Ad-hoc networks}

A wireless ad hoc network is a decentralized type of wireless network. The network is ad hoc because it does not rely on a preexisting infrastructure, such as routers in wired networks or access points in managed (infrastructure) wireless networks. Instead, each node participates in routing by forwarding data to other nodes, and so the determination of which nodes forward data is made dynamically based on the network connectivity. In addition to the classic routing, ad hoc networks can use flooding for forwarding the data. Since a centralized wired infrastructure is not required in ad-hoc networks, many medical devices can communicate with each other by routing traffic from source to destination through multiple hops [57]. Thus, ad-hoc networks are perfect alternative solution for emergency scenarios when either the existing communication system fails or there are no basic infrastructures in rural remote areas [58].

\section{Wireless sensor networks}

A wireless sensor network (WSN) consists of spatially distributed autonomous sensors to monitor physical or environmental conditions, such as temperature, sound, pressure, etc. and 
to cooperatively pass their data through the network to a main location. WSN technology is considered as one of the most important research areas in computer networks and eHealthcare application industries [21]. In e-Healthcare area, lots of medical applications are developed by working through WSN's in order to improve existing healthcare and monitoring services especially for the elderly and children [21]. Among all benefits, remote monitoring is the main thing for pervasive healthcare system. With remote monitoring, it becomes easier to monitor elder people's conditions to give timely response when emergency happens, also the children will be taken care of in a safer way when they are alone at home. All these tasks can be done without disrupting the patients' normal life.

\subsection{Potential challenges in design and implementation}

\section{Power allocation}

Conventional noise can be overcome by increasing transmit power, but it introduces interference. Therefore, in both centralized and ad hoc networks, if each device only uses the minimum required transmission power, the overall system capacity can be maximized while the interference caused to other devices is minimized [59]. The increased use of low cost Wi-Fi mesh networks makes it possible for some of the eHealthcare applications that require high transmission bandwidth to use Wi-Fi. A Wi-Fi mesh network operates on a multihop basis without the need for high transmission power. However, the delay increases and throughput degrades if there are many hops in between the users and gateway [54].

Authors in [60] describe a medium access control (MAC) protocol designed for wireless sensor networks used in e-Healthcare applications, with a goal of minimizing power consumption. The approach focuses on improving collision avoidance and the use of centrally controlled time-slot allocation to sensor nodes. Whereas in [30] for minimizing the power consumption of patient nodes, a simple algorithm is proposed for duty cycling to be implemented in the MAC sublayer of the Zigbee network. The authors conclude that precise duty 
cycling is the key to optimize network lifetime, considering other factors such as routing method and optimizing router locations.

\section{Resource management}

In [39], it is mentioned that tele-medicine and electronic health devices might not have packets to transmit all the time. WBASN's (as an example for WPAN's) usually are consisted of non-identical devices in terms of data rate or packet size. For low-power operations in such networks, IEEE 802.15.4 MAC is a widely used medium access protocol. It supports guaranteed time slot (GTS) allocation which offers scheduled transmissions and therefore saves the energy spent on carrier sensing otherwise. For the operation of IEEE 802.15.4 MAC in beacon enabled mode, this model considers the protocol parameters including idle time, GTS transmission mode, variable back-off window size and low data rate mode.

In OFDMA systems, several adjacent sub-carriers are usually grouped together to form a sub-channel. Authors in [61] investigate radio resource management (RRM) based on subchannels and for best effort services in the OFDMA downlink. They propose a novel subchannel allocation algorithm to both consider frequency selectivity and multi-user diversity gains in OFDMA networks. For RRM of multi-user OFDMA systems, RRM algorithms are categorized into two types: margin-adaptive and rate-adaptive. The objective of the former is to minimize the overall transmit power, with constraints on user's data rate, whereas the objective of the latter is to maximize the throughput of the system, having constraints on the total transmit power and bandwidth.

Resource Management is more challenging in heterogenous networks. While various network applications are being generated to function over heterogenous network resources within different management domains, their demands are dynamic. These demands include request for dedicated high bandwidth. Policy-based resource management and service provisioning technologies can be used to address these kind of demands [62]. There is an increase in the number of users of high performance applications which require network services that are 
not available on best effort infrastructures. These network requirements are usually referred to as "deterministic" network services. They have a guaranteed service level which usually includes a minimum bandwidth and an ability to specify loss rate, jitter, latency and other parameters.

\section{QoS support}

Quality of service (QoS) control mechanism is necessary to meet the minimum requirements of a variety of healthcare services which are delivered over networks in conjunction with other commercial traffic (e.g., voice calls, streaming multimedia, and Internet traffic) [41]. Thus, a QoS establishment is needed for applications of e-healthcare services to request the required QoS level and adapt to the real QoS offered by the network in reality. With a better QoS mechanism, less packet delay and losses result upon network congestion. For example, a TCP application may not sustain the same level of QoS as a UDP application because UDP offers one-way guaranteeing, while TCP generates a reverse acknowledgement (ACK) flow in addition to a data flow. QoS has the ability to offer different priorities to different applications, users, or data flows when the network capacity is insufficient.

Among many services providing QoS, Differentiated Service (DS) and Integrated Service (IS) are two promising ones. Both of them are Better-than-Best-Effort network services offering multiple levels of QoS. DS is a packet-based priority service, offering premium and assured services to meet differentiated requirements of e-healthcare applications. IS is a flow-based reservation service, providing controlled-load and guaranteed services to support delay intolerant services such as real-time service (e.g., tele-consultation services involving transmission of patient physiological parameters in emergency situations [41]).

In e-health or tele-medicine services over wireless medical networks, QoS provisioning is needed because of the strict requirements and real-time nature of medical applications. E-healthcare networks are supposed to operate 24 hours a day with no interruption. In medical network, delay is a very important factor, since medical communications should be 
timely to save critical patients' lives in most cases [55].

\section{Emerging technologies}

1. EMI mitigation. In [63] authors describe femtocell base stations as fully userdeployable which lead to reduced infrastructure and maintenance costs with positive impact on QoS. They identify interference as an important challenge which arises from mass deployment of femtocells. This directly affects capacity and achievable data rates. The solutions might vary depending on the technology used in physical layer. Cases of interference include co-channel deployment, adjacent channel deployment, and downlink and uplink interference [64]. Sometimes many femtocells and their as-

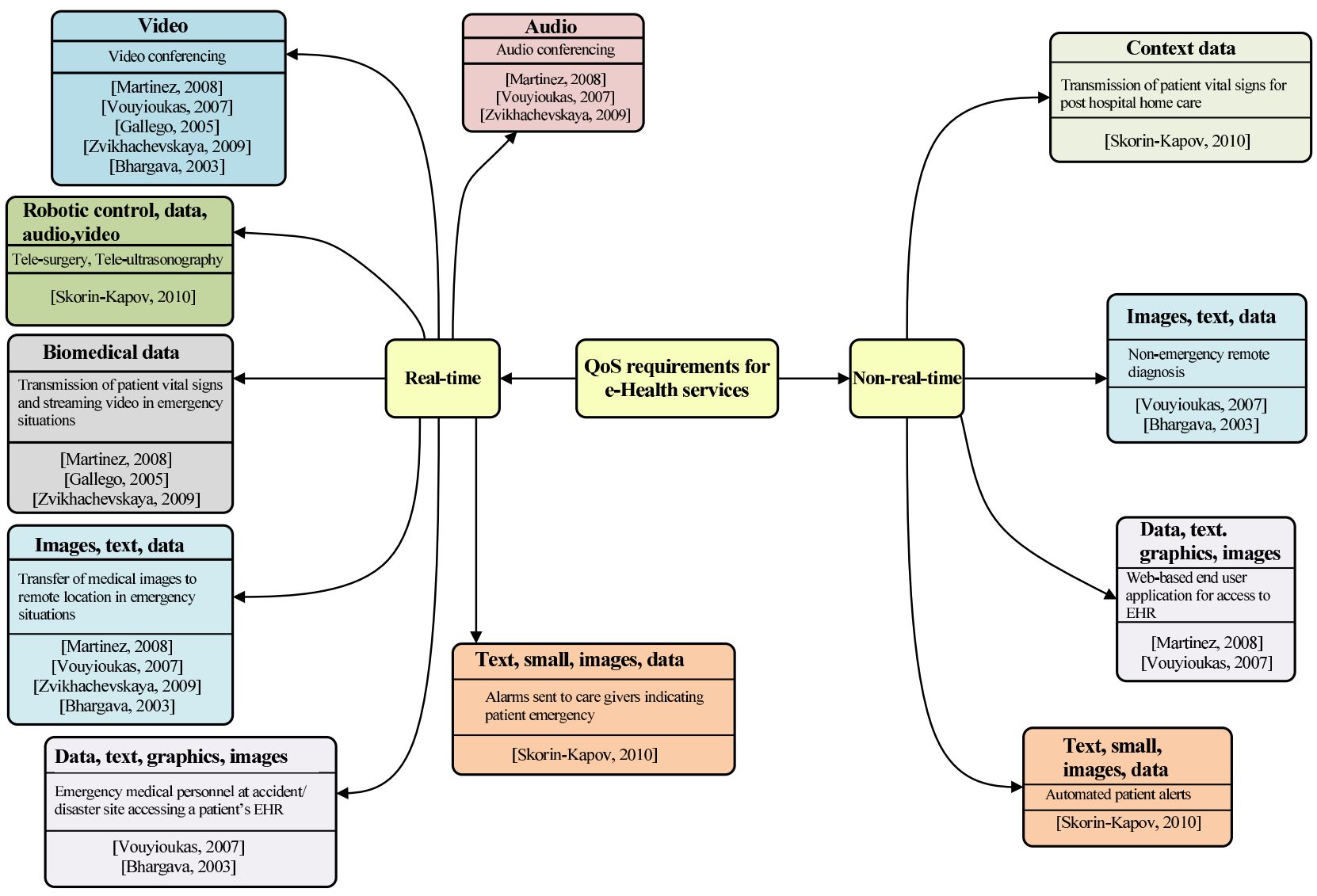

Figure 3.4: QoS requirements for e-Health services 
sociated macrocell use the same portion of spectrum, these can cause interruptions to each other [63]. Authors of [63] introduce different interference management techniques and categorize them as interference cancelation, interference avoidance and distributed interference management.

Interference cancelation includes schemes that reduce or cancel the interference at receiver, after the signal is received. Authors in [59] describe techniques that demodulate/decode the received signal and use the resulting information along with channel estimates to cancel the received interference from the received signal. In [63], authors indicate that interference avoidance is achieved by incorporating intelligence into femtocell access points. As these access points face different interference challenges depending on their location and the environment, it is hard to have a centralized control in such a scenario. Therefore, femtocell access point should be smart enough to cope with different situations and make sure that user receives a good quality service.

Distributed interference management schemes are suggested in different recent research due to impracticality of centralized techniques in larger networks. Femtocells have limited knowledge about the global femtocell network and providing sufficient information to femtocells through the backhaul network causes congestion on the network. Thus becomes obvious the importance of distributed schemes [63]. An appealing topic in [65] is about different interference management approaches and names them as cooperation among macrocell base stations (MeNBs) and femtocell base stations (HeNBs), exchange of information in groups of home evolved node Bs (path loss, location, etc.), intelligent spectrum access, etc.

2. Cognitive radio. As described in section 2.2 , cognitive radio is recent wireless concept that gives transceivers the ability to analyze the current channel state and alter some of their transmission parameters to optimize their operation and channel access. This technology deals with spectrum sensing, opportunistic channel access, radio resource 
management, interference mitigation, and other topics.

One of the examples where this concept is used is two-tier networks where macrocell and femtocell networks co-exist. Femtocell is designed to improve the data rate of end users along with reducing costs of network operator and improving capacity. However, interference might occur between femtocell users and macrocell users. In [66], the authors propose a single framework for cooperative spectrum sensing of cognitive radio networks which addresses the interference problem described.

Femtocells became popular in the field of wireless technology and deployed in many medical environment. Meanwhile, cognitive radio based on distributed spectrum sensing can be merged with femtocell for interference mitigation. Thus, the femtocells with cognitive functionalities are called cognitive femtocells [63], which makes a femtocell network intelligent enough to offer better indoor coverage and capacity. A cognitive femtocell can utilize the spectrum efficiently with the property of opportunistic spectrum access property, as well as avoiding interference by its ability to recognize an interference signature which can be used for femtocell to select certain channels that are not having interference problem [63].

\subsection{Chapter summary}

The improvements in computing and communication systems have had great influence in different aspect of our lives. One of the most important fields relates to healthcare. Trustworthiness of sensors, electronic devices, networks and wireless communications have reached to an extent that they can replace traditional legacy systems. Therefore, the term e-Healthcare is introduced. Regardless of all tremendous advances in this field, there still remains unexplored aspects of it especially considering the challenges. In this chapter, we discussed different elements of e-Healthcare, introduced wireless transmission technologies and standards that are used in e-Healthcare, presented the QoS requirements of each service, and 
discussed the potential challenges in power allocation, scheduling, meeting the QoS needs, and confronting the electromagnetic interference. Pervasive and continuous health monitoring is one of the most widely utilized services all over the world. It specifically provides the elderly and chronically ill patients with continuous and seamless health monitoring, benefiting both them and the caretakers.

In this chapter, power allocation, QoS support, EMI mitigation and cognitive radio are partially covered. The topology of a single floor in a hospital and wireless network environment are described in chapter 4 . In this environment, we formulate a multi-objective optimization problem as described in chapter 5. In the last chapter, we exploit our proposed branch-and-bound algorithm to solve our problem using OPTItoolbox in order to obtain the final solution and results. 


\section{Chapter 4}

\section{System Model and Assumptions}

\subsection{Hospital environment}

We consider a hospital building with $F(\geq 1)$ floors and multiple service areas (SA's) in each floor. A service area is defined as a region of a floor where wireless users and devices operate. The size, orientation and location of service areas totally depend on the building size and floor layout. Therefore, it is useful and interesting to have an inclusive deployment planning scheme for large hospital buildings. Users are distributed randomly within a service area. Figure 4.1 shows a typical multi-storey hospital building with wireless devices. In order to collect data from users (wireless transmitters), central processing points (CPP's) are installed in each floor. There can be more than one CPP in each floor. However, one service area can only be covered by one CPP whereas a CPP can cover multiple service areas.

Finally, CPP's can be connected to a gateway device that facilitates the transmission of data to remote locations. This device can be a gateway or a router capable of handling large amounts of data, such as medical images. The connection between CPP's and the gateway can be hard wired, with 802.3af $(\mathrm{PoE})$ enabled on the ports of the gateway device. Usually when there are several CPP's working in the same group, a central controller is used. This has various benefits including facilitating management and configuring of CPP's, 


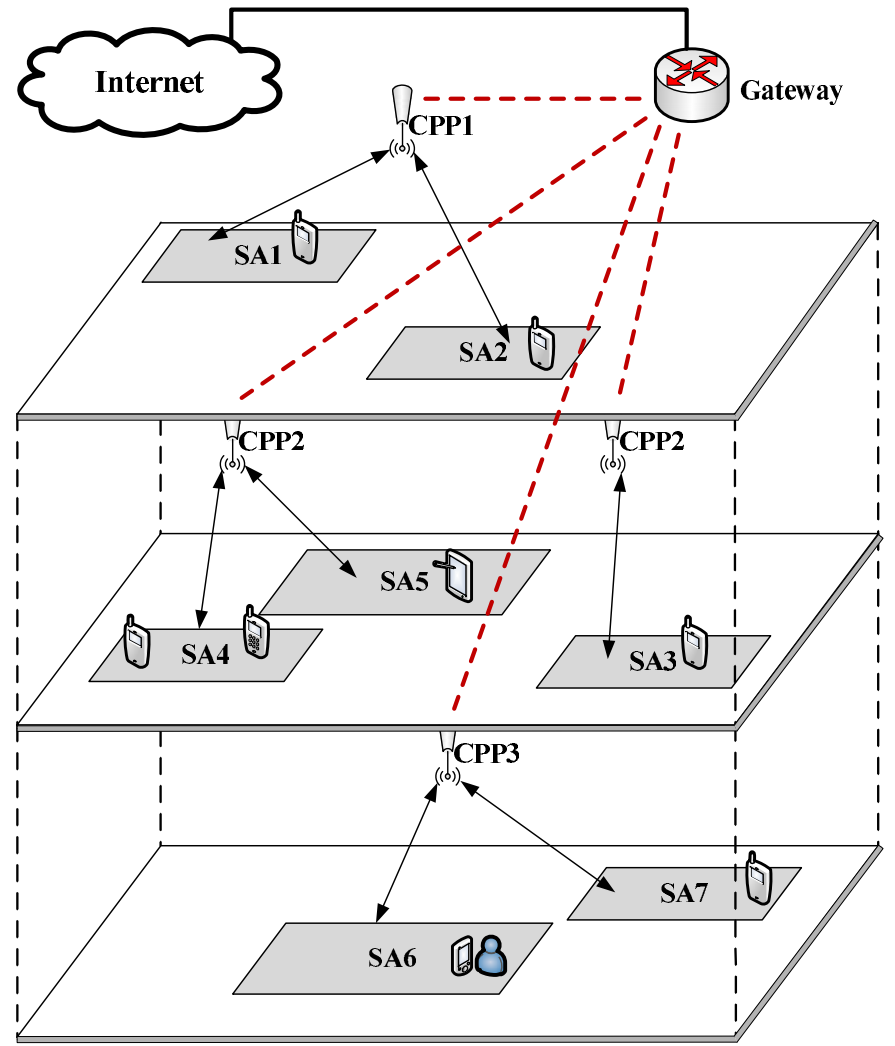

Figure 4.1: CPP placement in multi-storey buildings.

automatic adjustment of channels, authentication, security features and the output power of the transmitters. The latter is very important in preventing adjancent-channel interference. This concept is briefly described in section 6.2.2.

For simplicity, we assume the service areas to be of equal size, each having an area of a quarter of a floor's area. To protect medical devices from electromagnetic interference (EMI), protected areas are defined in the middle of SA's. EMI sensitive medical devices are only allowed to operate in these areas. Electronic components of such devices show performance deterioration when exposed to electromagnetic field. The effects include automatic shutdown, automatic restart, altered monitored results, etc. Examples of these devices are defibrillators, incubators (as life-supporting devices) infusion pumps and ECG monitors (as 
non-life-supporting devices) [6]. In Chapter 5, we will discuss how we implement interference avoidance in our modeling for devices in protected areas. The goal of our proposition is to minimize the EMI in terms of the intensity of the electronic field in protected areas.

Users in Figure 4.1 are considered to have devices with wireless transmission, for example telemetry devices. CPP's control the wireless transmission of users and collect their data in uplink communication. CPP placement optimization in hospitals is important as it has a direct impact on the transmission power of the users. In other words, we want to optimize uplink power of users and network coverage in hospitals at the same time. In our problem, we aim to determine the coordinates of CPP's (i.e., $x, y$ and $z$ ) and also meet the quality of service (QoS) requirement of each user. This model considers floor attenuation factor (FAF) and CPP installation restrictions. To represent the users of service areas, our problem translates to minimizing the worst user's transmission power (a min-max problem).

We want to optimally deploy CPP's in a hospital building to minimize the worst user's transmission power in each SA under the QoS, EMI and assignment constraints. QoS is defined as satisfied data rate. This can be translated into the received power at the CPP for each user, to be greater than a minimum value. That is, the attenuated uplink power of users due to different obstacles in the building, wall or floor separation, and the distance to the assigned CPP, is considered as the received power at that CPP for the respective user. A path loss model will be introduced in chapter 5 . Table 4.1 contains the notations used in this text and their definition.

We consider a wireless network in one floor of a hospital, with $M$ CPP's and $N$ SA's. Each SA has $K$ users or wireless nodes that transmit data to their associated CPP. Therefore, in total there exist $\hat{K}=N \times K$ users in the hospital. $F$ denotes the number of floors in the hospital building which will be considered as 1 for most of the cases investigated in this thesis. The height of each floor is denoted by $h . \eta$ will be used to represent the average height of a user which means the height that users operate and transmit wirelessly.

As mentioned earlier, we consider uplink transmission power of users and an important 
Table 4.1: Notations

\begin{tabular}{|c|c|}
\hline Notation & Definition \\
\hline$M$ & Number of central processing points (CPP's) \\
\hline$N$ & Number of service areas (SA's) \\
\hline$K$ & Number of users in each SA \\
\hline$\hat{K}$ & Number of users in the whole building $(\hat{K}=N \times K)$ \\
\hline$F$ & Number of floors \\
\hline$D$ & $\begin{array}{l}\text { An } N \times M \text { matrix containing the distances between } \\
\mathrm{CPP} \text { 's and SAs }\end{array}$ \\
\hline$\left(u_{n}, v_{n}, w_{n}\right)$ & Coordinates of the center of the SA $n$ \\
\hline$\left(x_{m}, y_{m}, z_{m}\right)$ & Coordinates of CPP $m$ \\
\hline$h$ & The height of each floor \\
\hline$\eta$ & Average height of a user \\
\hline$\varepsilon$ & $\begin{array}{l}\text { Floor attenuation factor constant (depends on the envi- } \\
\text { ronment) }\end{array}$ \\
\hline$\alpha$ & Path loss exponent \\
\hline$E_{\max }$ & $\begin{array}{l}\text { Maximum allowed electric field from transmitters mea- } \\
\text { sured in the middle of protected area }\end{array}$ \\
\hline$p_{n}$ & $\begin{array}{l}\text { Transmission power of the user in } n^{t h} \text { SA that is furthest } \\
\text { away from its associated CPP }\end{array}$ \\
\hline$p_{\max }$ & The maximum transmission power limit of every user \\
\hline$\hat{p}$ & Minimum received power at CPP for all users \\
\hline$Z$ & $\begin{array}{l}\text { The constant from the free-space impedance of a pro- } \\
\text { tected device }\end{array}$ \\
\hline$I$ & $\begin{array}{l}\text { The immunity level of a device at the center of protected } \\
\text { area }\end{array}$ \\
\hline$\hat{d}_{n k}$ & $\begin{array}{l}\text { The distance between the } k^{t h} \text { user in } n^{t h} \text { SA from the } \\
\text { center of protected area }\end{array}$ \\
\hline$a_{n m}$ & An $N \times M$ association matrix where $a_{n m} \in\{0,1\}$ \\
\hline$\Omega\left(z_{m}, w_{n}\right)$ & $\begin{array}{l}\text { An indicator function: } \\
\qquad \Omega\left(z_{m}, w_{n}\right)=\left\{\begin{array}{l}\Omega_{0} \\
\Omega_{1}\end{array}\right.\end{array}$ \\
\hline$\Omega_{0}, \Omega_{1}$ & depending on the specific environment \\
\hline
\end{tabular}

parameter in this context is the distance between the transmitter and the CPP. The problem we define in chapter 5 is a min-max problem and we aim to minimize the maximum uplink transmission power from each SA. Hence, within an SA, only the worst user (the user with the maximum uplink power) will be of interest. In the channel model that will be described 
in section 4.3, the worst user is the user that is located the furthest from its associated CPP. Therefore, an $N \times M$ matrix, $D$, is defined that contains the distances between CPP's and the worst users of SA's.

For EMI calculation, another distance variable is needed because in this context, the worst user will be the closest user to the center of the protected area. Therefore, an $N \times K$ matrix, $\hat{d}_{n k}$, is defined which contains the distances between wireless users of the $n^{\text {th }} \mathrm{SA}$ and the center of the protected area of the same SA. In our formulation, referring to SA's is done by addressing the center of them. We denote the coordinates of the center of $n^{\text {th }} \mathrm{SA}$ by $\left(u_{n}, v_{n}, w_{n}\right)$. Also the location of $m^{t h}$ CPP is shown by $\left(x_{m}, y_{m}, z_{m}\right)$.

In Chapter 5, we discuss how to obtain the penetration loss between two adjacent floors. For this purpose $\varepsilon$ is defined to represent the floor attenuation constant which depends on the specific environment and is calculated according to the floor attenuation factor. In this context, $\alpha$ shows path loss exponent, that is, signal attenuation rate. We define $\Omega\left(z_{m}, w_{n}\right)$ as an indicator function whose value depends on $z_{m}$ and $w_{n}$. In Table 4.1, in definition of $\Omega\left(z_{m}, w_{n}\right)$, the parameters $\Omega_{0}$ and $\Omega_{1}$ are constants that depend on the environment. In order to show the association of SA's to CPP's, we define $a_{n m}$ to be an $N \times M$ matrix where its elements are either 0 or 1 . A "1" at the location $[n, m]$ represents the association of $n^{\text {th }}$ SA to the $m^{\text {th }}$ CPP.

As mentioned earlier, according to our channel model, the worst user is the user located the furthest from its associated CPP. Therefore, minimizing the uplink transmission power of all the users within an SA, boils down to minimizing the worst user's power within that SA. We define $p_{n}$ as a vector, containing the worst user's transmission power in each SA. $p_{\max }$ sets a limit for uplink transmission power of all users whereas $\hat{p}$ is the minimum received power at a $\mathrm{CPP}$ for all of its wireless users, defined according to QoS requirements. In dealing with EMI, the immunity level of medical devices is an important factor. We denote this parameter with $I$, which has a unit of volts/meter. If we have $K$ users in a service area, the maximum allowed electric field measured in the middle of the protected area caused by 
each wireless transmitter is defined as $E_{\max }$ and is equal to:

$$
E_{\max }=\frac{I}{K}
$$

Obviously, if we have only one user in each service area, $E_{\max }$ and $I$ will be identical. In this context, we also define $Z$, as the constant from the free-space impedance of a protected device. To learn more about how we assign the value of $Z$, refer to Section 6.1.

\subsection{Transceiver model}

In our model, each CPP has its own frequency band not to interfere with adjacent CPP's. SA's operate on orthogonal channels to avoid co-channel interference. Therefore, when two or more SA's are connected to one CPP, transmissions from one SA will not harm those of other SA's connected to the same CPP. Within SA's, multiple access schemes are deployed for user transmissions so that multiple users can transmit at the same time to one CPP. EMI immunity level of devices operating in the protected area is known in terms of electric field. $p_{n}$ is the transmission power of a user in the $n^{\text {th }} \mathrm{SA}$ that is located in the furthest distance from its associated CPP. That is, we consider the worst wireless user in terms of channel gain with the CPP. This concept is shown in Figure 4.2a. This figure illustrates a typical $\mathrm{SA}$ in a horizontal plane in which, point $\mathrm{B}$ is the furthest away from the $m^{\text {th }} \mathrm{CPP}$.

We consider the furthest point because the transmission power of a user in this location would be the maximum transmission power of all users in that SA in order to accommodate QoS. We define a guard area around the protected area according to the maximum transmission power limit of users $\left(p_{\max }\right)$. Therefore, even if a user is on the border of guard area and transmits with its maximum allowed transmission power, there is not going to be

electromagnetic interference (EMI) on devices inside the protected area due to the electric field from transmitters in SA.

Figure $4.2 \mathrm{~b}$ illustrates the vertical projection of a typical hospital layout. To satisfy the QoS requirements of users, we define $\hat{p}$ as the minimum power received at a CPP from each 


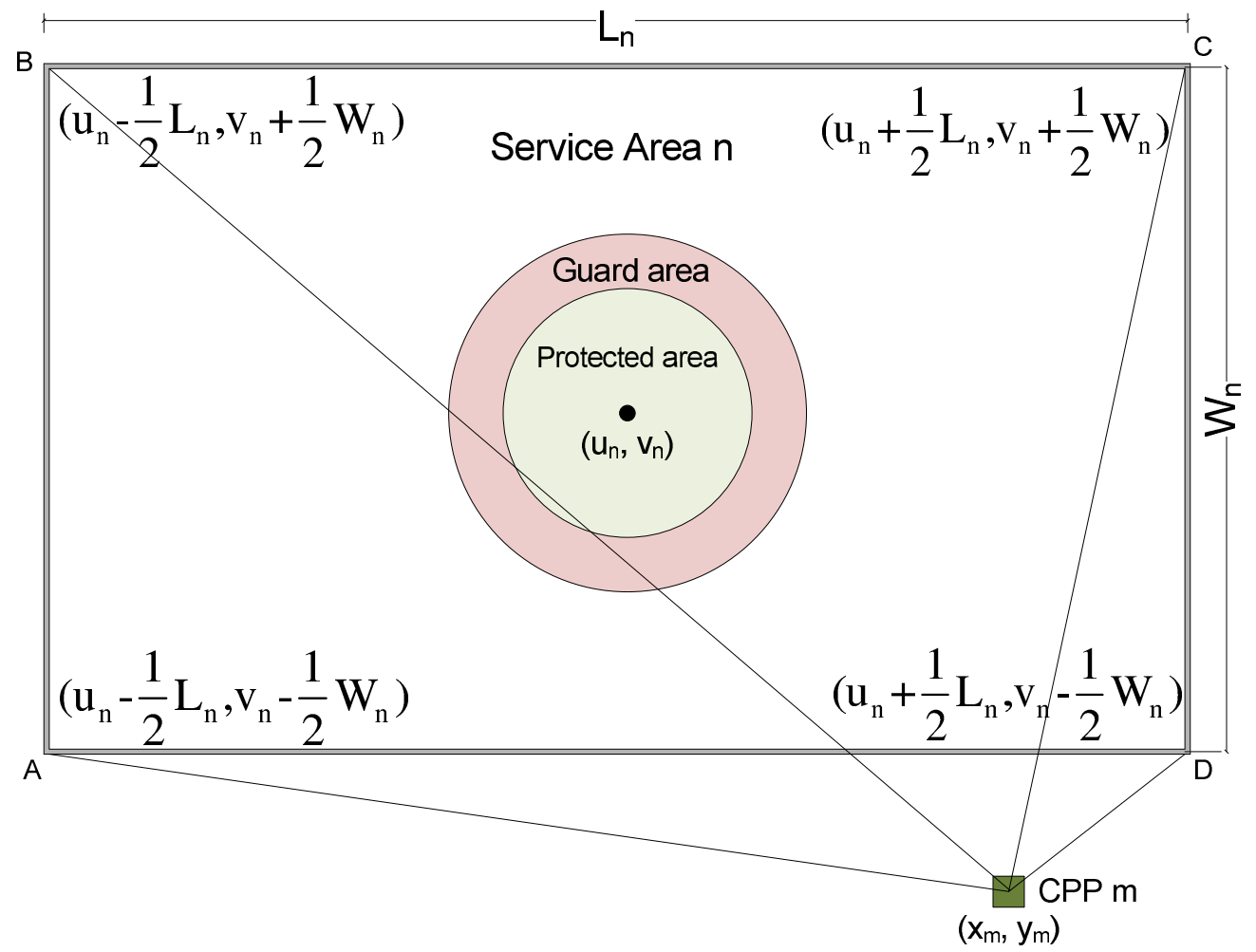

(a) Horizontal projection of a typical SA and its associated CPP.

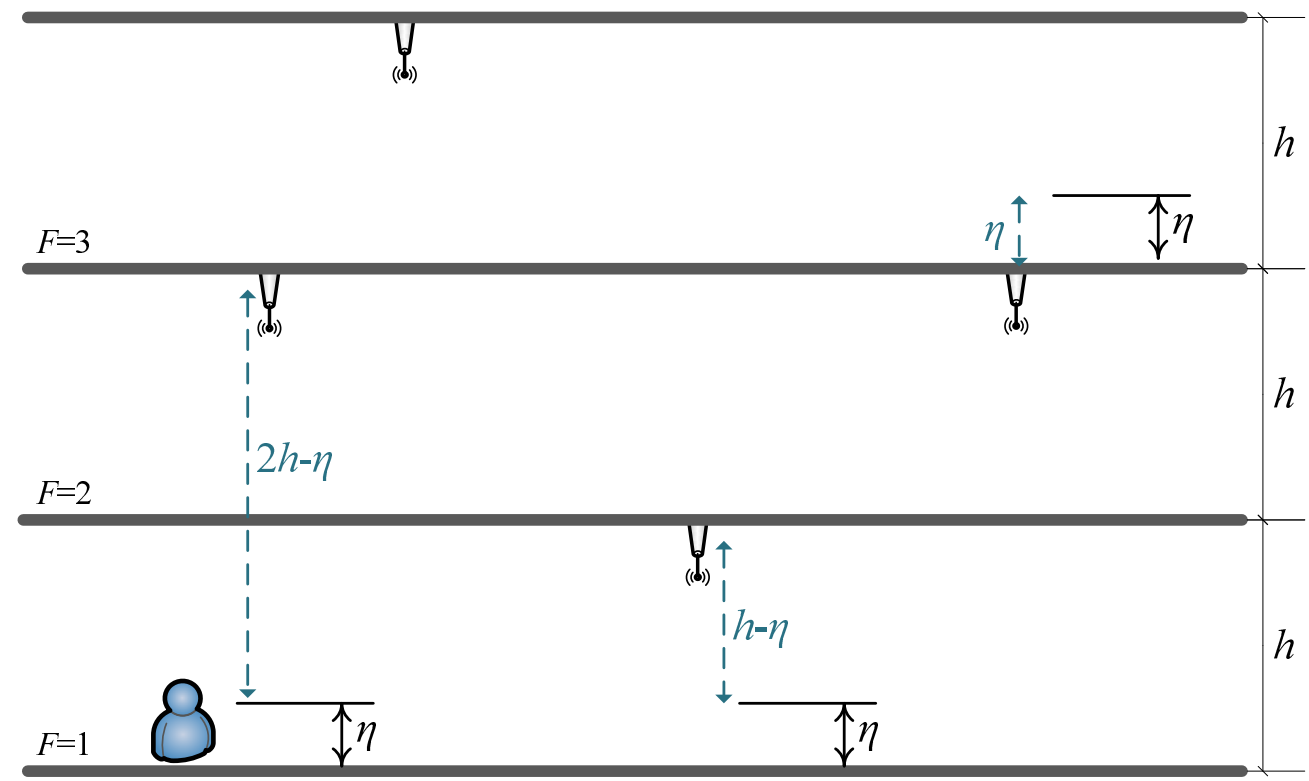

(b) Vertical projection of the multi-storey building shown in Figure 4.1.

Figure 4.2: Horizontal and vertical views of an SA and hospital building, respectively 
user. If the received power at a CPP for a user is greater than this value, then the SINR requirement of users is met. That is: $S I N R_{k} \geq S I N R_{r e q}, \quad \forall k$.

\subsection{Channel model}

One of the key components to channel response is path loss. When modeling path loss for a channel, different factors should be considered with the most important being the attenuation factor. A very simple channel can be considered as the free space line of sight when there are no obstacles between transmitter and receiver. In this case, transmitted signal attenuates before getting to the receiver due to energy loss. In theory, this loss is proportional to the inverse of square of the distance. However, as will be introduced in Chapter 5, we consider a path loss exponent, $\alpha$, because in practice power attenuates more quickly, typically proportional to inverse of 4th power of the distance [67].

SA's operate in non-overlapping channels meaning that they use FDMA for instance and do not cause interference to each other's transmission. In WLAN, there are eight FDMA channels. If we are using IEEE 802.11a, there are 23 non-overlapping channels in the $5 \mathrm{GHz}$ band that adjacent transmitters can exploit to transfer simultaneously without interfering each other. If IEEE $802.11 \mathrm{~b} / \mathrm{g}$ is used, we can only have three non-overlapping channels which operate in $2.4 \mathrm{GHz}$ frequency band. Within SA's, simultaneous user transmissions can be achieved using orthogonal channels (such as using time, frequency or code). The AWGN noise is negligible.

\subsection{Chapter summary}

In this chapter, we defined our system model and explained various parts of it. Starting with the structure of a hospital building with a single floor that will be used in our simulation, we introduced floor layout, different elements and areas within the floor, and data collection

model of the wireless network. Also, the transceiver model was explained in details including 
transmission power of wireless users, immunity level of medical devices and other factors affecting the transmission of data. Then, we described communication environment and channel model with a focus on our path loss scheme and the wireless technology considered for our problem. Finally, the parameters and notations used in this thesis were all explained within this chapter, preparing the reader for formulation of our problem which will follow in the next chapter. 


\section{Chapter 5}

\section{Problem Formulation}

Our focus in this thesis is on jointly optimizing the power consumption of user nodes and CPP deployment, considering that the QoS requirements must be met. Also EMI on EMIsensitive medical devices has to be minimized. Our assumption is that each transmitter is assigned an orthogonal frequency band. Channel assignment is a vast discussion and is beyond the scope of this thesis work. According to our system model described in the previous chapter, following discussion, results in the formulation of our problem.

\subsection{Initial formulation}

For optimizing the life time of nodes, we need to minimize the maximum transmission power originating from the service areas: $\operatorname{Min}\left\{\operatorname{Max}\left\{p_{n}\right\}\right\}$ This can be translated into two statements with the help of an auxiliary variable, $\lambda$ :

$$
\text { Minimize } \lambda
$$

s.t.

$$
\lambda \geq p_{n}, \quad \forall n .
$$

As mentioned, $p_{n}$ represents transmission power of the user in $n^{\text {th }}$ SA that is furthest 
away from its associated CPP (compared to point B in Figure 4.2a). Users have transmission power limit that we denote as:

$$
0 \leq p_{n} \leq p_{\max }, \quad \forall n
$$

where $p_{\max }$ represents the maximum transmission power limit of each user. In this context, we assume that this limit is the same for all users for simplicity. In Section 6.1, we will discuss proper value assignment for $p_{\max }$.

For path loss modeling, we need to consider the hospital building environment. Due to varying interior partitions and different physical characteristic of the partitions, it is impossible to use a general path loss model for all types of buildings. In this thesis, we deploy the path loss model used in [5]. In this model, $\alpha$ represents the path loss exponent and shows the rate at which the signal attenuates. Different values of $\alpha$ can be used to model different buildings. Some of these values can be found in Table 4.3 in [68]. To provide an estimate of indoor path loss, this exponent is combined with a floor attenuation factor which depends on the environment and number of floors between transmitter and receiver. Average values for floor attenuation factor in $\mathrm{dB}$ for one, two, three and four floor separation can be observed in Table 4.4 in [68]. We use $\varepsilon$ to represent the floor attenuation constant which depends on the specific environment and is obtained according to the floor attenuation factor. Under this path loss model, we can calculate the received power $\left(p_{R_{n}}\right)$ of users at their associated CPP as follows:

$$
p_{R_{n}}=\frac{p_{n}}{\Omega\left(z_{m}, w_{n}\right) D^{\alpha} \varepsilon^{\left|z_{m}-w_{n}\right|}}, \quad \forall n, m
$$

where $\Omega\left(z_{m}, w_{n}\right)$ is an indicator function whose value depends on $z_{m}$ and $w_{n}$ and is defined as:

$$
\Omega\left(z_{m}, w_{n}\right)= \begin{cases}\Omega_{0}, & z_{m}=w_{n} \\ \Omega_{1}, & z_{m} \neq w_{n}\end{cases}
$$


where $\Omega_{0}$ and $\Omega_{1}$ are constants that depend on the environment, $z_{m}$ is the floor number that the $m^{\text {th }} \mathrm{CPP}$ is located in, and $w_{n}$ is the floor number that the $n^{\text {th }} \mathrm{SA}$ is located in. $\varepsilon$ is the floor attenuation constant which also depends on the environment and $D$ is the distance between the corresponding CPP and SA. (see [5], Appendix A for how to calculate $\varepsilon, \Omega_{0}$ and $\left.\Omega_{1}\right)$.

In order to accommodate QoS for users at a certain data rate, the received power level of users at their associated CPP must be more than a certain threshold. We show this threshold with $\hat{p}$ and consider it to be the same for all users. Thus, we can reformulate the received power equation as:

$$
\frac{p_{n}}{a_{n m} \Omega\left(z_{m}, w_{n}\right) D^{\alpha} \varepsilon^{\left|z_{m}-w_{n}\right|}} \geq \hat{p}, \quad \forall n, m
$$

where $a_{n m}$ is an association matrix with its elements being either 1 or 0 . This matrix shows the association of SA's to CPP's. Comparing this constraint to the received power equation in (5.4), it can be easily verified that, if $a_{n m}=1$ (i.e. if $m^{\text {th }}$ CPP is associated with the $n^{\text {th }}$ $\mathrm{SA}$ ), then this constraint is identical to the received power equation with a minor alteration. On the other hand, for when $a_{n m}=0$, after further reformulation, it can be shown that this constraint reduces to $p_{n} \geq 0$, which is trivially valid. Usually, the association model between wireless transmitters and receivers is based on the distance between them. However, in commercial buildings, such as hospitals, the association model becomes more complicated and the closest CPP is not necessarily the best. The reason is that there can be one floor separation between the SA and the closest CPP and due to penetration loss through one floor separation, the signal quality might be very low at the SA. Therefore, instead of defining a specific association rule, we let our optimization problem decide about the association between SA's and CPP's. In Chapter 6, we will demonstrate this with an example, where the SA's and CPP's are located in different floors. As not more than one CPP can be 
associated with an SA at a time, we can formulate association constraint as:

$$
\sum_{m=1}^{M} a_{n m}=1, \quad \forall n
$$

According to Figure 4.2a, we can calculate the horizontal distance between an SA and its associated CPP. To ensure that a CPP covers a whole SA, we define the horizontal distance as a straight line between the CPP and the furthest point of the SA. It can be shown that the projection of the horizontal distance on $x$-axis and $y$-axis are $\left|x_{m}-u_{n}\right|+\frac{1}{2} L_{n}$ and $\left|y_{m}-v_{n}\right|+\frac{1}{2} W_{n}$, respectively.

In Figure 4.2b, a typical installation of the CPP's is shown which is installing the CPP's on the ceiling. This is the most practical way of installing CPP's to avoid any probable obstacle. Also average height of users is demonstrated in this figure and denoted by $\eta$. This variable is defined in respect to the average human height. Assuming $h$ to be height of each floor, the vertical distance between a CPP and a user can be obtained from $\left|\left(z_{m}-w_{n}+1\right) h-\eta\right|$. This can be verified in Figure $4.2 \mathrm{~b}$.

With the help of the vertical distance and projections of horizontal distance on $x$-axis and $y$-axis, the distance between the $m^{\text {th }} \mathrm{CPP}$ and $n^{\text {th }} \mathrm{SA}$ can be computed using the following equation:

$$
\begin{array}{r}
D=\left[\left(\left|x_{m}-u_{n}\right|+\frac{1}{2} L_{n}\right)^{2}+\left(\left|y_{m}-v_{n}\right|+\frac{1}{2} W_{n}\right)^{2}\right. \\
\left.+\left|\left(z_{m}-w_{n}+1\right) h-\eta\right|^{2}\right]^{\frac{1}{2}}, \quad \forall m, n,
\end{array}
$$

In order to implement interference avoidance in our modeling for devices in protected areas consider the following equation for calculating the electric field from transmitters [6], to the middle of a protected area:

$$
E=\frac{Z \sqrt{p_{n}}}{\hat{d}_{n k}}
$$

where $E$ is the electric field measured at the middle of the protected area and caused by 
the wireless transmitters, $Z$ is the constant from the free-space impedance of a protected device, and $\hat{d}_{n k}$ is the distance between the $k^{t h}$ user in the $n^{t h}$ SA from the center of the protected area. If we rewrite (5.8) as what follows, we both have converted this non-convex constraint to a convex constraint, and can set a maximum value for the electric field sensed in the middle of the protected area:

$$
E_{\max } \geq \frac{Z \sqrt{p_{n}}}{\hat{d}_{n k}}
$$

Further, we can rewrite (5.9) in terms of transmission power, to get a sense of a limit for uplink transmission power of users:

$$
0 \leq p_{n} \leq\left(\frac{E_{\max } \hat{d}_{n k}}{Z}\right)^{2}
$$

Based on the discussion we had in this section, we present the initial formulation of our problem as follows:

$$
\underset{x_{m}, y_{m}, z_{m}, p_{n}, a_{n m}}{\operatorname{Minimize}} \lambda
$$

s.t.

$$
\begin{aligned}
& \lambda \geq p_{n}, \quad \forall n, \\
& \frac{p_{n}}{a_{n m} \Omega\left(z_{m}, w_{n}\right) D^{\alpha} \varepsilon^{\left|z_{m}-w_{n}\right|}} \geq \hat{p}, \quad \forall n, m, \\
& \sum_{m=1}^{M} a_{n m}=1, \quad \forall n, \\
& 0 \leq p_{n} \leq\left(\frac{E_{\text {max }} \hat{d}_{n k}}{Z}\right)^{2},
\end{aligned}
$$

where

$$
D=\left[\left(\left|x_{m}-u_{n}\right|+\frac{1}{2} L_{n}\right)^{2}+\left(\left|y_{m}-v_{n}\right|+\frac{1}{2} W_{n}\right)^{2}+\left|\left(z_{m}-w_{n}+1\right) h-\eta\right|^{2}\right]^{\frac{1}{2}}, \quad \forall m, n .
$$




\subsection{Reformulation}

According to initial formulation, constraint (5.5) contains binary variable $a_{n m}$ and constraint (5.7) contains non-convex elements. Therefore, the problem defined in (5.11) is a mixed-integer non-convex programming problem which is generally NP-hard. Also the term $a_{n m} \Omega\left(z_{m}, w_{n}\right) D^{\alpha} \varepsilon^{\left|z_{m}-w_{n}\right|}$ in (5.5) makes this constraint a complex one. Following are the steps to transform this problem into an equivalent mixed integer convex problem.

Letting $\Delta \triangleq D$, we have:

$$
\Delta=\left(\left|x_{m}-u_{n}\right|+\frac{1}{2} L_{n}\right)^{2}+\left(\left|y_{m}-v_{n}\right|+\frac{1}{2} W_{n}\right)^{2}+\left|\left(z_{m}-w_{n}+1\right) h-\eta\right|^{2}
$$

Constraint (5.12) is equivalent to:

$$
\Delta \geq\left(\left|x_{m}-u_{n}\right|+\frac{1}{2} L_{n}\right)^{2}+\left(\left|y_{m}-v_{n}\right|+\frac{1}{2} W_{n}\right)^{2}+\left|\left(z_{m}-w_{n}+1\right) h-\eta\right|^{2}
$$

The inequality in (5.13) holds as an equality at an optimal solution. As a proof, suppose that for some $n$ and $m,(5.13)$ holds strictly as an inequality. By decreasing the corresponding values of $\Delta$ to make (5.13) hold as an equality, (5.13) and (5.12) will be equivalent and the feasibility in the revised constraint is maintained. It can be easily verified that (5.13) is a convex constraint in contrary to (5.12). However, the absolute values on the right hand side of constraint (5.13) are not differentiable which makes it difficult to implement an algorithm to solve the equation. In order to simplify it, we assume that $X_{n m} \triangleq\left|x_{m}-u_{n}\right|$ and $Y_{n m} \triangleq\left|y_{m}-v_{n}\right|$. Thus, (5.13) can be rewritten as following set of constraints:

$$
\begin{array}{r}
\left(X_{n m}+\frac{1}{2} L_{n}\right)^{2}+\left(Y_{n m}+\frac{1}{2} W_{n}\right)^{2}+\mid\left(h z_{m}-\left[\left(w_{n}-1\right) h+\eta\right]\right)^{2}-\Delta \leq 0 \\
X_{n m}=\left|x_{m}-u_{n}\right|, \\
Y_{n m}=\left|y_{m}-v_{n}\right| .
\end{array}
$$

It can be observed that in (5.14), the first constraint is convex; however, the second and 
third constraints require absolute value operations. To further linearize them we can replace them with their equivalent terms: $X_{n m} \geq\left|x_{m}-u_{n}\right|$ and $Y_{n m} \geq\left|y_{m}-v_{n}\right|$. By reformatting these constraints we get:

$$
\begin{array}{r}
\left(X_{n m}+\frac{1}{2} L_{n}\right)^{2}+\left(Y_{n m}+\frac{1}{2} W_{n}\right)^{2}+\mid\left(h z_{m}-\left[\left(w_{n}-1\right) h+\eta\right]\right)^{2}-\Delta \leq 0 \\
u_{n} \geq x_{m}-X_{n m}, \\
-u_{n} \geq-x_{m}-X_{n m}, \\
v_{n} \geq y_{m}-Y_{n m}, \\
-v_{n} \geq-y_{m}-Y_{n m} .
\end{array}
$$

Now we reformulate the received power constraint which is the most complex of all. According to (5.5) and (5.12), we can consider the following change of variable as the first step:

$$
\frac{p_{n}}{a_{n m} \Omega\left(z_{m}, w_{n}\right) \Delta^{\frac{\alpha}{2}} \varepsilon^{\left|z_{m}-w_{n}\right|}} \geq \hat{p}
$$

Then, by rearranging the variables we get:

$$
a_{n m} \Omega\left(z_{m}, w_{n}\right) \Delta^{\frac{\alpha}{2}} \varepsilon^{\left|z_{m}-w_{n}\right|} \hat{p}-p_{n} \leq 0,
$$

Constraint (5.17) can be linearized with respect to binary variable $a_{n m}$, thus the following equivalent representation is introduced:

$$
\Omega\left(z_{m}, w_{n}\right) \Delta^{\frac{\alpha}{2}} \varepsilon^{\left|z_{m}-w_{n}\right|} \hat{p}-\left(1-a_{n m}\right) U_{n m}-p_{n} \leq 0
$$

where $U_{n m}$ is an upper bound for $\Omega\left(z_{m}, w_{n}\right) \Delta^{\frac{\alpha}{2}} \varepsilon^{\left|z_{m}-w_{n}\right|} \hat{p}, z_{m}$ and $w_{n}$ are the floor number of the $m^{\text {th }} \mathrm{CPP}$ and $n^{\text {th }} \mathrm{SA}$, respectively and $\hat{p}$ is the minimum transmission power of wireless transmitters. In order to verify the equivalency of (5.17) and (5.18), we can replace $a_{n m}$ by its possible values. If $a_{n m}=0$, we have $\Omega\left(z_{m}, w_{n}\right) \Delta^{\frac{\alpha}{2}} \varepsilon^{\left|z_{m}-w_{n}\right|} \hat{p}-U_{n m} \leq 0 \leq p_{n}$ which is a trivially valid statement. Also, if $a_{n m}=1,(5.17)$ and (5.18) are equivalent. In (5.18), one 
value for the upper bound $U_{n m}$ would be obtained from:

$$
U_{n m} \triangleq \hat{p} \max \left\{\Omega_{0}, \Omega_{1}\right\} \Delta_{U}^{\frac{\alpha}{2}} \varepsilon^{\max \left\{w_{n}-1, F-w_{n}\right\}},
$$

where $\Delta_{U}$ is an upper bound for $\Delta$. A valid value for $\Delta_{U}$ can be obtained as follows:

$$
\begin{aligned}
\Delta_{U} & =\max \left\{\left(u_{n}+\frac{1}{2} L_{n}\right)^{2},\left(x_{\max }-u_{n}+\frac{1}{2} L_{n}\right)^{2}\right\} \\
& +\max \left\{\left(v_{n}+\frac{1}{2} W_{n}\right)^{2},\left(y_{\max }-v_{n}+\frac{1}{2} W_{n}\right)^{2}\right\} \\
& +\max \left\{\left(\left(2-w_{n}\right) h-\eta\right)^{2},\left(\left(F-w_{n}+1\right) h-\eta\right)^{2}\right\} .
\end{aligned}
$$

To linearize the terms $\Delta^{\frac{\alpha}{2}}$ and $\varepsilon^{\left|z_{m}-w_{n}\right|}$ in (5.18), we define two variables, $\tau$ and $\xi$, where $\tau=\Delta^{\frac{\alpha}{2}}$ and $\xi=\varepsilon^{\left|z_{m}-w_{n}\right|}$. Therefore, we can rewrite (5.18) as the following three constraints:

$$
\begin{array}{r}
\Omega\left(z_{m}, w_{n}\right) \tau \xi \hat{p}-\left(1-a_{n m}\right) U_{n m}-p_{n} \leq 0, \\
\tau=\Delta^{\frac{\alpha}{2}}, \\
\xi=\varepsilon^{\left|z_{m}-w_{n}\right|} .
\end{array}
$$

According to discussions in this chapter, the non-convex constraint $\tau=\Delta^{\frac{\alpha}{2}}$ can be convexified as:

$$
\tau \geq \Delta^{\frac{\alpha}{2}}
$$

where, as mentioned for (5.5), we have $\alpha \geq 2$ and therefore (5.22) is convex.

Next, to linearize and convexify the term $\xi=\varepsilon^{\left|z_{m}-w_{n}\right|}$ in (5.21), we take the following step. Starting with $z_{m}$ that has integer values in interval $\{1, \ldots, F\}$, with $F$ being the number of floors, this phrase can be replaced by the following binary representation:

$$
z_{m}=\sum_{f=1}^{F} f \gamma_{f}^{m}, \quad \forall m
$$


where $\gamma_{f}^{m} \in\{0,1\}$ and the following holds:

$$
\sum_{f=1}^{F} \gamma_{f}^{m}=1, \quad \forall m
$$

Now, $\xi=\varepsilon^{\left|z_{m}-w_{n}\right|}$ can be replaced by its equivalent linear constraint:

$$
\xi=\sum_{f=1}^{F} \gamma_{f}^{m} \varepsilon^{\left|f-w_{n}\right|}, \quad \forall m, n,
$$

Using (5.25), we can convert (5.21) to a constraint that only involves binary variables:

$$
\Omega\left(z_{m}, w_{n}\right) \tau_{n m} \hat{p} \sum_{f=1}^{F} \gamma_{f}^{m} \varepsilon^{\left|f-w_{n}\right|}-\left(1-a_{n m}\right) U_{n m}-p_{n} \leq 0, \quad \forall m, n,
$$

According to the definition of $\Omega\left(z_{m}, w_{n}\right)$ in Table 4.1, the constraint in (5.26) can be modified as:

$$
\Omega_{1} \tau_{n m} \hat{p} \sum_{f=1, f \neq w_{n}}^{F} \gamma_{f}^{m} \varepsilon^{\left|f-w_{n}\right|}+\Omega_{0} \tau_{n m} \hat{p} \gamma_{w_{n}}^{m}-\left(1-a_{n m}\right) U_{n m}-p_{n} \leq 0, \quad \forall m, n,
$$

Thus following is the equivalent reformulation for our initial problem defined in (5.11):

$$
\underset{x_{m}, y_{m}, z_{m}, p_{n}, a_{n m}}{\operatorname{Minimize}} \lambda
$$

s.t.

$$
\begin{aligned}
& \lambda \geq p_{n}, \quad \forall m, n, \\
& \Omega_{1} \tau_{n m} \hat{p} \sum_{f=1, f \neq w_{n}}^{F} \gamma_{f}^{m} \varepsilon^{\left|f-w_{n}\right|}+\Omega_{0} \tau_{n m} \hat{p} \gamma_{w_{n}}^{m}-\left(1-a_{n m}\right) U_{n m}-p_{n} \leq 0, \quad \forall m, n, \\
& \tau_{n m} \geq \Delta_{n m}^{\frac{\alpha}{2}}, \quad \forall m, n,
\end{aligned}
$$




$$
\begin{aligned}
& z_{m}=\sum_{f=1}^{F} f \gamma_{f}^{m}, \quad \forall m, \\
& \sum_{f=1}^{F} \gamma_{f}^{m}=1, \quad \forall m, \\
& \sum_{m=1}^{M} a_{n m}=1, \quad \forall n, \\
& 0 \leq p_{n} \leq p_{\max }, \quad \forall m, \\
& 0 \leq p_{n} \leq\left(\frac{E_{\max } \hat{d}_{n k}}{Z}\right)^{2}, \quad \forall m \\
& \left(X_{n m}+\frac{1}{2} L_{n}\right)^{2}+\left(Y_{n m}+\frac{1}{2} W_{n}\right)^{2}+\left(h z_{m}-\left[\left(w_{n}-1\right) h+\eta\right]\right)^{2}-\Delta_{n m} \leq 0, \quad \forall m, n, \\
& u_{n} \geq x_{m}-X_{n m}, \quad \forall m, n, \\
& -u_{n} \geq-x_{m}-X_{n m}, \quad \forall m, n, \\
& v_{n} \geq y_{m}-Y_{n m}, \quad \forall m, n, \\
& -v_{n} \geq-y_{m}-Y_{n m} \quad \forall m, n \text {. }
\end{aligned}
$$

\subsection{Branch-and-bound algorithm}

According to our formulation in (5.28-5.41) and based on what was discussed earlier, our problem is a convex mixed integer non-linear programming (MINLP) problem. One of the practical algorithms for solving MINLP problems is the branch and bound algorithm. In this section, we will have a review on branch and bound algorithm and our proposed variation of it used in this thesis work.

In MATLAB, there are toolboxes that incorporate branch and bound algorithm. In order to solve our optimization problem, we employ OPTimizition Interface Toolbox (OPTItoolbox) which is basically based on branch and bound, and outer approximation algorithms. OPTItoolbox can be used for constructing and solving linear, nonlinear, continuous and discrete optimization problems. A range of open source and academic solvers are supplied 
inside the toolbox. Figure 5.1 demonstrates the steps of the simulation in MATLAB using a flowchart.

With OPTItoolbox, we can construct optimization problems and solve them using a range of supplied solvers. OPTItoolbox is capable of automatically detecting the problem type being solved and chooses the best solver available for the case, using powerful open source solvers such as IPOPT, SCIP and NOMAD. In our case, we use OPTItoolbox to solve our optimazition problem which is a convex MINLP. To solve convex MINLP, OPTItoolbox uses BONMIN (the default MINLP solver), which stands for Basic Open Source Nonlinear Mixed Integer Programming. BONMIN is an experimental open-source $\mathrm{C}++$ code for solving general MINLP problems. It solves smooth, twice differentiable, mixed integer nonlinear programs. BONMIN uses IPOPT for solving relaxed problems and CBC as the mixed integer solver. It also contains a number of algorithms for convex MINLPs [69].

As mentioned earlier, for solving MINLP, we choose a branch and bound (BB) approach, which is an efficient way to solve MINLP. BB is a classical algorithm for solving MINLP. One ingredient is a candidate solution, i.e., a point feasible for MINLP. It is updated as soon as a feasible point with a lower objective value is encountered. As such, the candidate solutions yield a decreasing sequence of upper bounds on the optimal objective value of MINLP. A candidate solution might not yet be known at the beginning of the algorithm. Also, the algorithm maintains a set of restrictions of MINLP, for which some of the lower (respectively, upper) bounds on integer variables are increased (respectively, decreased) from their original values. The set of restrictions is chosen so that if there exists a better candidate than the current one, it is feasible for at least one of the restrictions in the set.

Branch-and-bound algorithms are used extensively for mixed-integer non-linear programming (MINLP) models. The basic algorithm is applicable to model non-linear functions where a non-linear solver needs to evaluate the relaxed sub-problems. In [70], a standard branch-and-bound algorithm for MINLP problems with integer variables is introduced.

Algorithm 1 demonstrates our approach in employing branch-and-bound method. In 


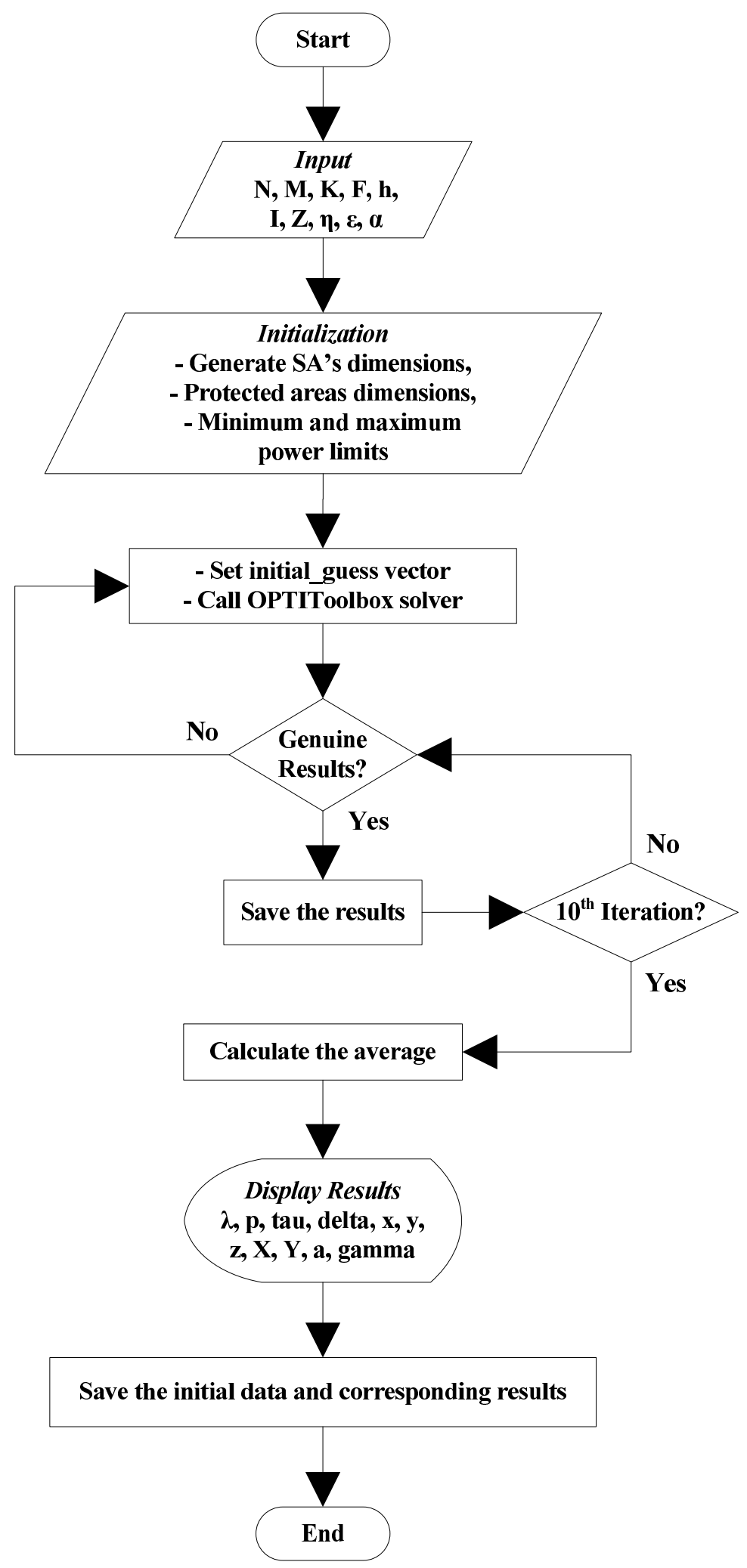

Figure 5.1: Flowchart of simulation process in MATLAB using OPTIToolbox. 
this algorithm, $L B$ is lower bound of objective function, $U B$ is the upper bound of objective function and $o b j$ is the value for objective function. In our problem, the objective function is $\lambda$ and its value is between 0 and $p_{\max }$.

We denote our decision variables with $x_{k}$ 's where $k \in 1, \ldots, \Theta$ and $\Theta$ is the number of our decision variables which depends on $N$, number of SA's, $F$, number of floors in the hospital, and $M$, number of CPP's. Decision variables, although initialized when enumerating the initial guess vector, are determined inside the program loop. They include $p_{n}$, transmission power for wireless users, $\tau$ and $\Delta$, both auxiliary variables for distance between a CPP and its associated SA's, $x_{m}, y_{m}$ and $z_{m}$, coordinates of CPP's, $X_{n m}$ and $Y_{n m}$, auxiliary variables for the projection of horizontal distance on $x$-axis and $y$-axis, $a_{n m}$, the association matrix and $\gamma_{f}^{m}$, auxiliary variable for the floor number of CPP's.

\subsection{Chapter summary}

In this chapter, we described the formulation of our problem of transmission power and EMI minimization, CPP deployment optimization, and CPP-to-SA association. We represented an intuitive initial formulation and moved onto a robust reformulation of a multi-objective mixed-integer non-linear programming (MINLP) problem. In our multi-objective formulation, the objective function consists of maximum transmission power of wireless users that has to be minimized. However, there are several decision variables that affect the value of the objective function. These decision variables are also determined within the program, so as to confirm the constraints and yield an optimal global result.

The algorithm we used for solving the aforementioned MINLP problem, is a branch-andbound algorithm and was explained at the end of this chapter. The programming is done in MATLAB and results are obtained using the solvers available in OPTIToolbox. In the next chapter, we will present the numerical results of the simulation. 


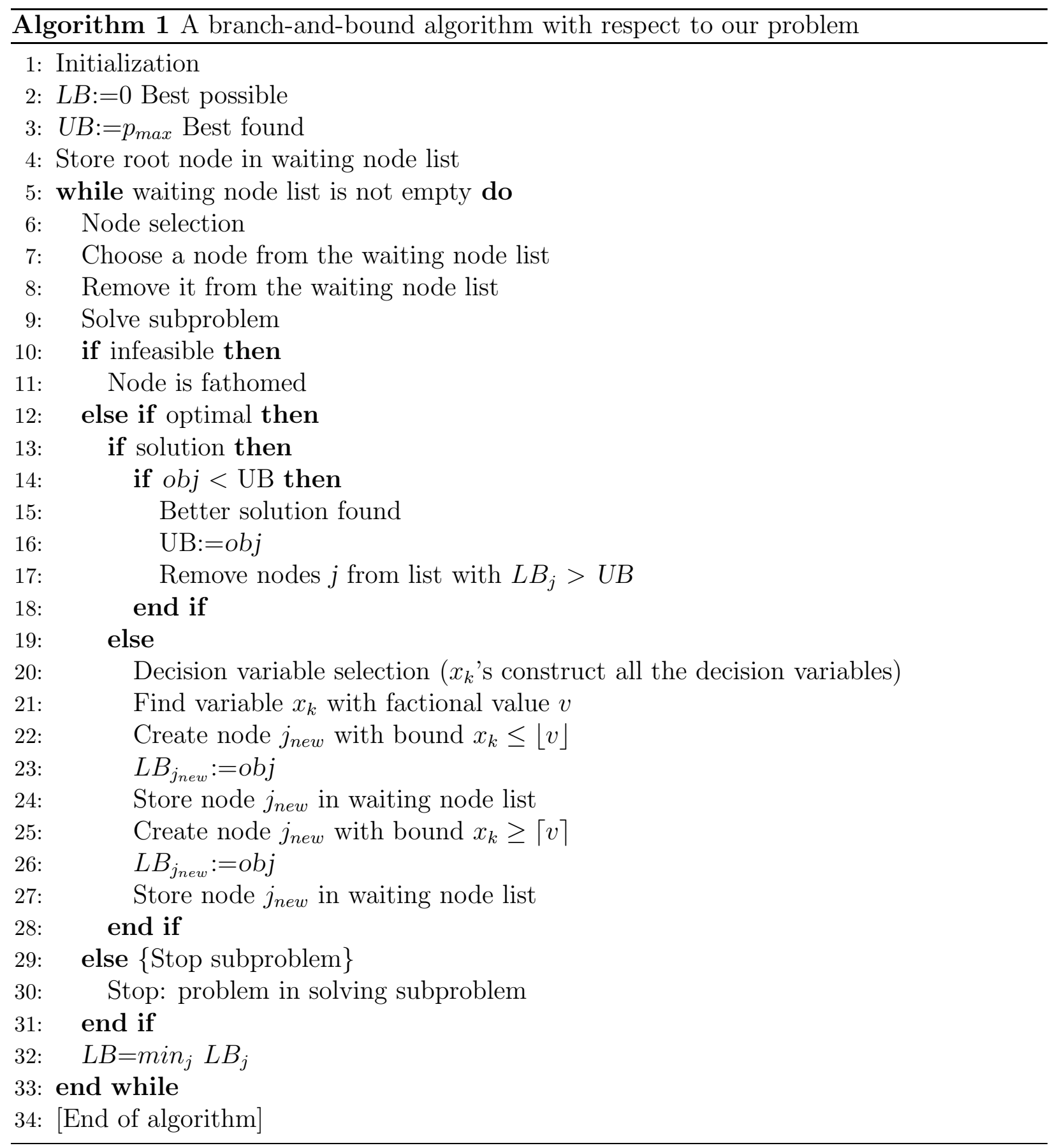




\section{Chapter 6}

\section{Experimental Evaluation and Simulation Results}

In this chapter, we conduct numerical simulation and analysis of the proposed formulation and solution, using MATLAB and the OPTItoolbox. Initially the simulation setup will be explained along with value assignment to different variables. Afterwards, the results for running our MATLAB program to solve the objective function will be presented and analyzed.

\subsection{Simulation setup}

Starting with more intuitive layouts of the building and SA's, initially we consider having one SA on the first floor of a two-storey hospital building with a CPP installed on the ceiling of the first floor. The length, width and height of each floor are 60, 40 and 3 meters respectively. Later on, the effect of the floor height on the users' transmission power will be investigated.

Emphasizing on the visibility and intuitiveness of the results, for all the SA's, the length and width will be considered the same at the beginning. Each SA will be 30 meters long and 20 meters wide. Therefore, we can have 4 SA's of the same size on each floor. It will be 
assumed that each floor has only one user which requires the maximum transmission power of all the users within that SA. It is then translated to the user located the furthest away from its associated CPP. For more detailed explanation, refer to Chapter 5. Thus, from this point on, transmission power of users in an SA will be referred to as the transmission power of that SA.

By running the program in MATLAB we are aiming to get the coordinations of the $\mathrm{CPP}(\mathrm{s})$, as well as the transmission power of each SA and association of the SA's to CPP's. In the path loss model described in Chapter 5, in order to accommodate QoS, the received power of an SA at its associated CPP should not be less than $-100 \mathrm{dBm}$. To reflect this, we assume that $\hat{p}=10^{-10} \mathrm{~mW}$. The maximum power level for IEEE 802.11g-based radio devices is $30 \mathrm{~mW}$ when using OFDM. OFDM is a commonly considered physical layer technology in modern wireless communication. Therefore, we let $p_{\max }$ be $30 \mathrm{~mW}$ during the whole simulation process.

On the other hand, considering EMI avoidance limits the maximum transmission power of SA's as well. The maximum electric field experienced by devices in the protected area should not exceed their EMI immunity level. This electric field is imposed by the users within the SA and this field is at its maximum when a transmitting user is on the border of the guarded area. A guarded area is considered to have a radius of 3 meters whereas the protected area within has that of 2 meters. With this size of the guarded area, it makes sense for our simulation to have an immunity level of $I=1$ for sensitive devices in the center of protected area. IEC $60601-1-2$ specifies $Z$ to be equal to $23 \sqrt{\Omega}$ for life-supporting devices, between the frequencies $800 \mathrm{MHz}$ and $2.5 \mathrm{GHz}$ [6]. Under these values, the transmission power of users is further limited to a maximum of $17 \mathrm{~mW}$. Thus if we relax the EMI constraint for a specific room, the value of $p_{\max }=30 \mathrm{~mW}$ will be taken into account.

We let path loss exponent, $\alpha$, to be 4 for all cases. Also the first floor attenuation factor for the floor separation is set to $16.2 \mathrm{~dB}$ according to the average values in [68], Table 4.5. 


\subsection{Numerical results}

\subsubsection{One CPP}

The program is then run for different number of SA's within a floor when there is one CPP available. Figure 6.1 demonstrates the maximum transmission power of SA's. According to this figure, the transmission power of SA's increase when the number of the SA's increase for a single CPP. The power is minimized to $1.2 \mathrm{~mW}$ for 4 SA's.

Figure 6.2 shows a 3D demonstration of the CPP placement and SA positions.

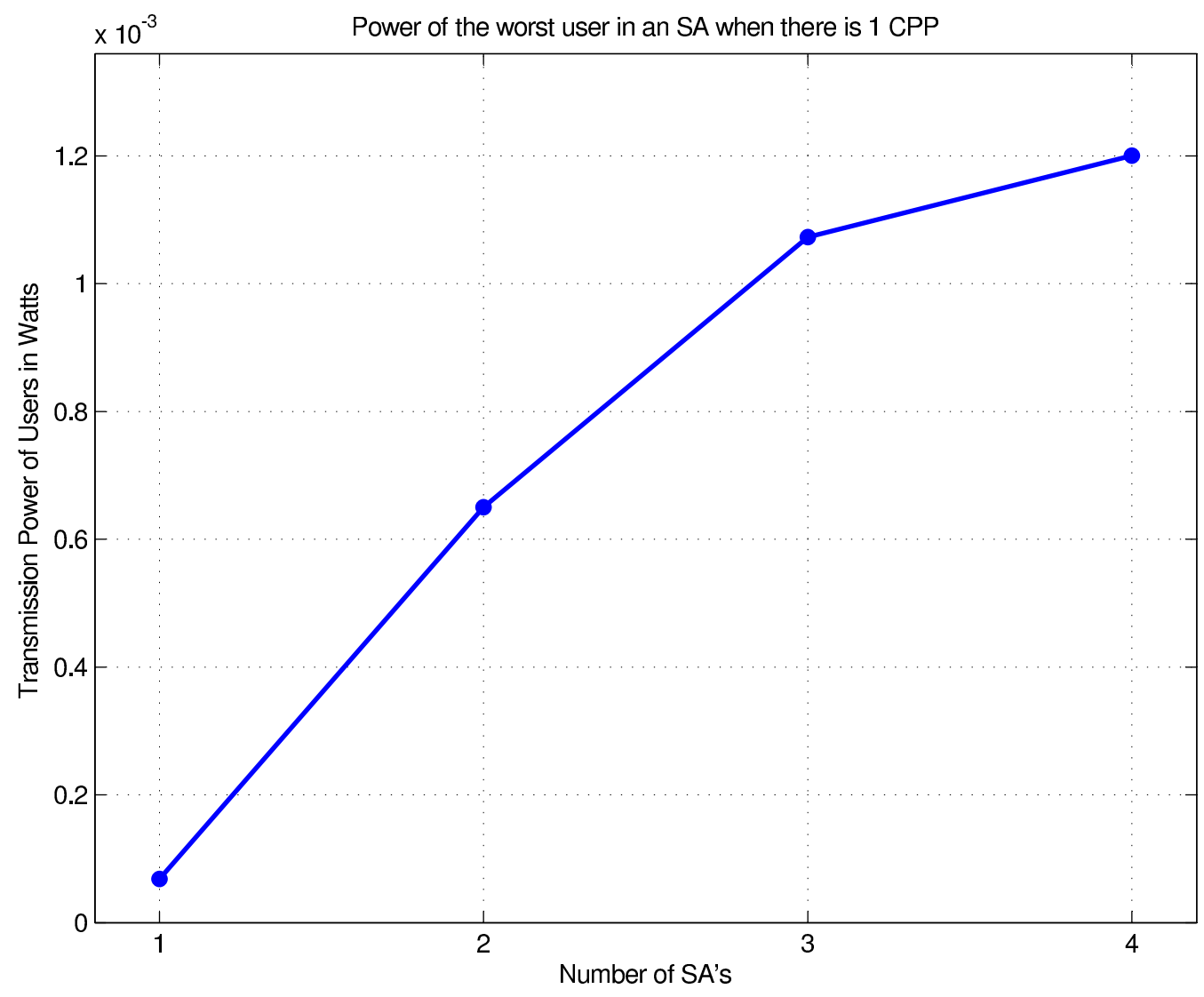

Figure 6.1: Transmission power required by the user of and SA, located the furthest from its associated CPP for different number of SA's. This power satisfies the QoS requirement and EMI avoidance in the protected area. 


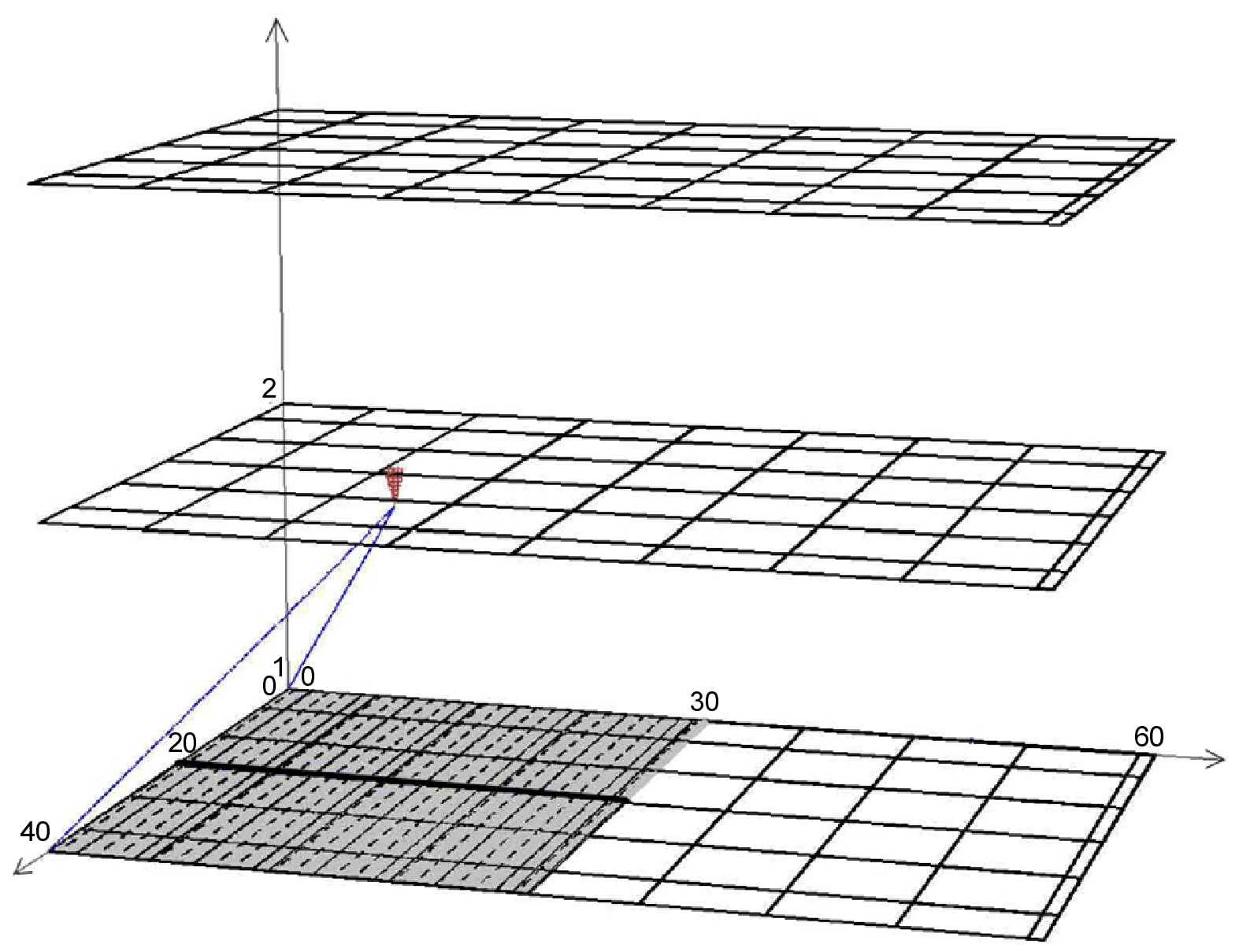

Figure 6.2: A 3D model of when a CPP is assigned to 2 SA's within the same floor.

Table 6.1 contains more details and numbers regarding the results for when we have one CPP. The results of this thesis work is obtained under the channel model described in section 4.3. It considers a free space line of sight environment, when there are no obstacles between transmitter and receiver. If the service areas are surrounded by walls, transmission power, for example, will be much higher. The attenuation factor of a typical interior wall at hospitals is $7 \mathrm{~dB}$ in average, compared to that of a concrete wall which is $12 \mathrm{~dB}$ in average. This will impose deployment of more CPP's within a floor in order to accommodate the power limits of users and the QoS.

The consumption power highly depends on the distance of a transmitting user from its associated CPP. In this part, we examine the effect of the floor height on the transmission 
power of an SA. As can be seen in Figure 6.3, power has increased for all the cases when we changed the height from 2.5 to 3 and 3 to 3.5 meters. The cases considered in this experiment were identical to the cases explained for Figure 6.1.

Next we investigate the effect of penetration loss through one floor. We assumed a single service area with a length of 30 meters and width of 20 meters on the second floor. In the program, we forced one CPP to be on the first floor and the other on the second floor. With

Table 6.1: Numerical results for when there is one CPP for different number of SA's

\begin{tabular}{|l|c|c|c|c|}
\hline & 1 SA & 2 SA's & 3 SA's & 4 SA's \\
\hline$\lambda$ & $68.28 \mu \mathrm{W}$ & $649.9 \mu \mathrm{W}$ & $1.073 \mathrm{~mW}$ & $1.201 \mathrm{~mW}$ \\
\hline Coordinates of CPP (x,y) & $(15,10)$ & $(15,20)$ & $(27.36,18.24)$ & $(30,20)$ \\
\hline Association & $\{1\}$ & $\{1,1\}$ & $\{1,1,1\}$ & $\{1,1,1,1\}$ \\
\hline
\end{tabular}

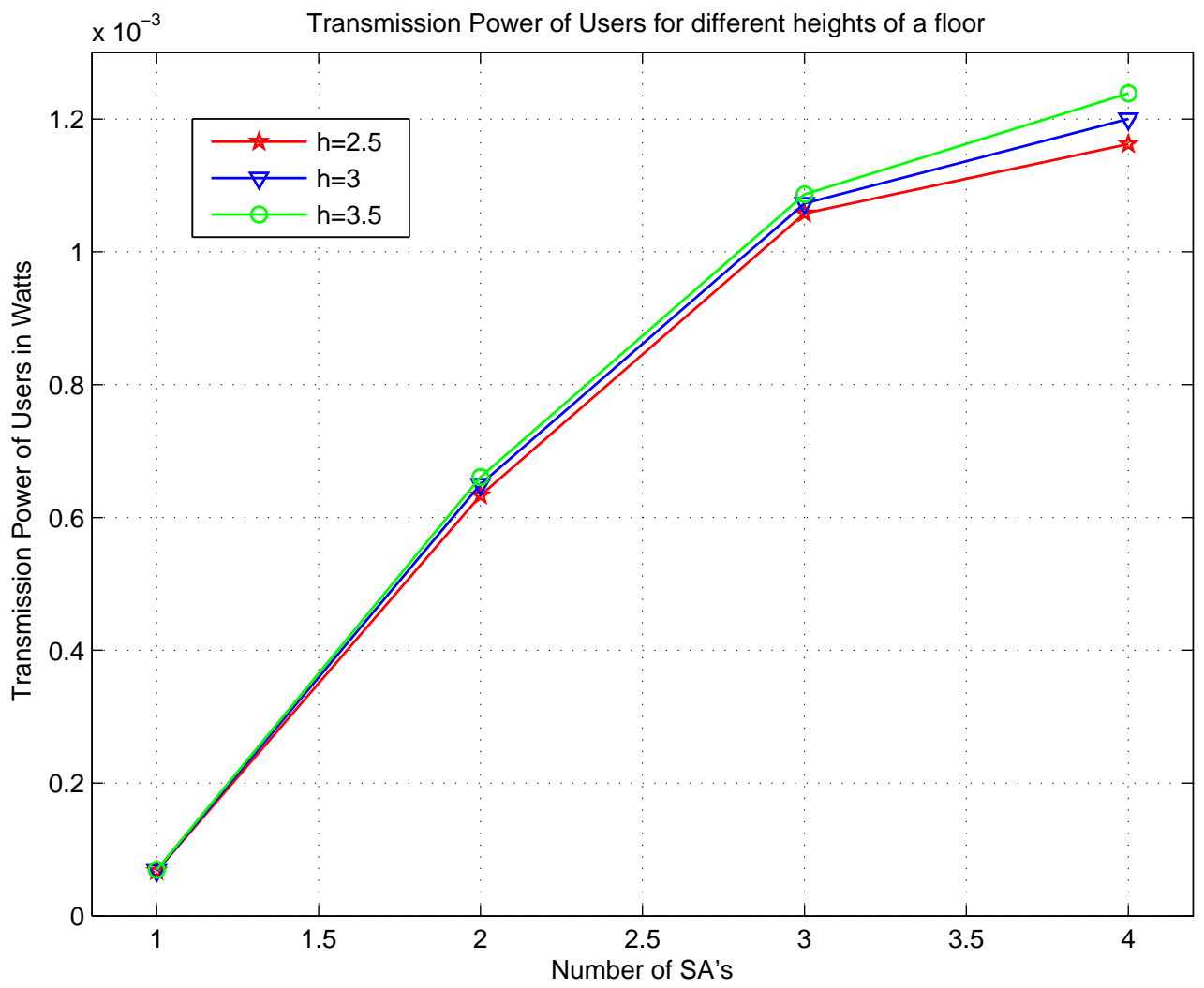

Figure 6.3: Effect of height of the floor on maximum transmission power required by the user of an SA, located at the furthest point from its associated CPP, for different number of SA's. 
the floor attenuation factor for one floor separation being equal to $16.2 \mathrm{~dB}$, the simulation results returned the assignment of the $\mathrm{CPP}$ on the second floor to the SA. When the value of the floor attenuation factor was reduced to $1 \mathrm{~dB}$, the results showed a different assignment. As the distance between the SA and the CPP right below it on the first floor, is less than that of the $\mathrm{CPP}$ on the second floor, the SA will have an association to the $\mathrm{CPP}$ on the ceiling of the first floor.

In case we have one $\mathrm{SA}$ in the second floor and force it to connect to the CPP right below it on the ceiling of the first floor, the power consumption significantly increases. The results gave us a value of $2.493 \mathrm{~mW}$ for this SA's transmission power which is significantly higher than other users' transmission power. This value is also confirmed with the following

equation: Loss in $\mathrm{dB}=10 \log \left(\frac{P_{t}}{P_{r}}\right)$ where $P_{t}$ and $P_{r}$ represent the transmission power and the received power, respectively.

A remarkable characteristic of our program is providing immunity assurance for EMI sensitive medical devices. As a reminder, the electric field around medical devices which is caused by transmitters within SA's can be calculated using the following equation [6]: $E=\frac{Z \sqrt{p_{n}}}{\hat{d}_{n k}}$ which can be resorted to find a limit for $p_{n}$ when $E$ changes. Medical devices have different sensitivity threshold. Some of these devices are life-supporting with a lower threshold and some are non-life-supporting with a greater threshold. The lower the immunity level of devices are, the lower the electric field around them should be. To address this, maximum transmission power limit of users is manipulated. If we need to accommodate highly sensitive devices, the power limit in our program is further reduced.

\subsubsection{Two CPP's}

Next, we consider having two CPP's on a floor. For the case of two SA's at location $(0,0)$ and $(0,20)$, the results return the location of CPP's at $(15,10)$ and $(15,30)$ which is demonstrated in Figure 6.4. The transmission power for the SA's are recorded as $68.36 \mu \mathrm{W}$ and $68.37 \mu \mathrm{W}$. In Figure 6.5a the coverage of the CPP's and their overlapping areas for the previously 


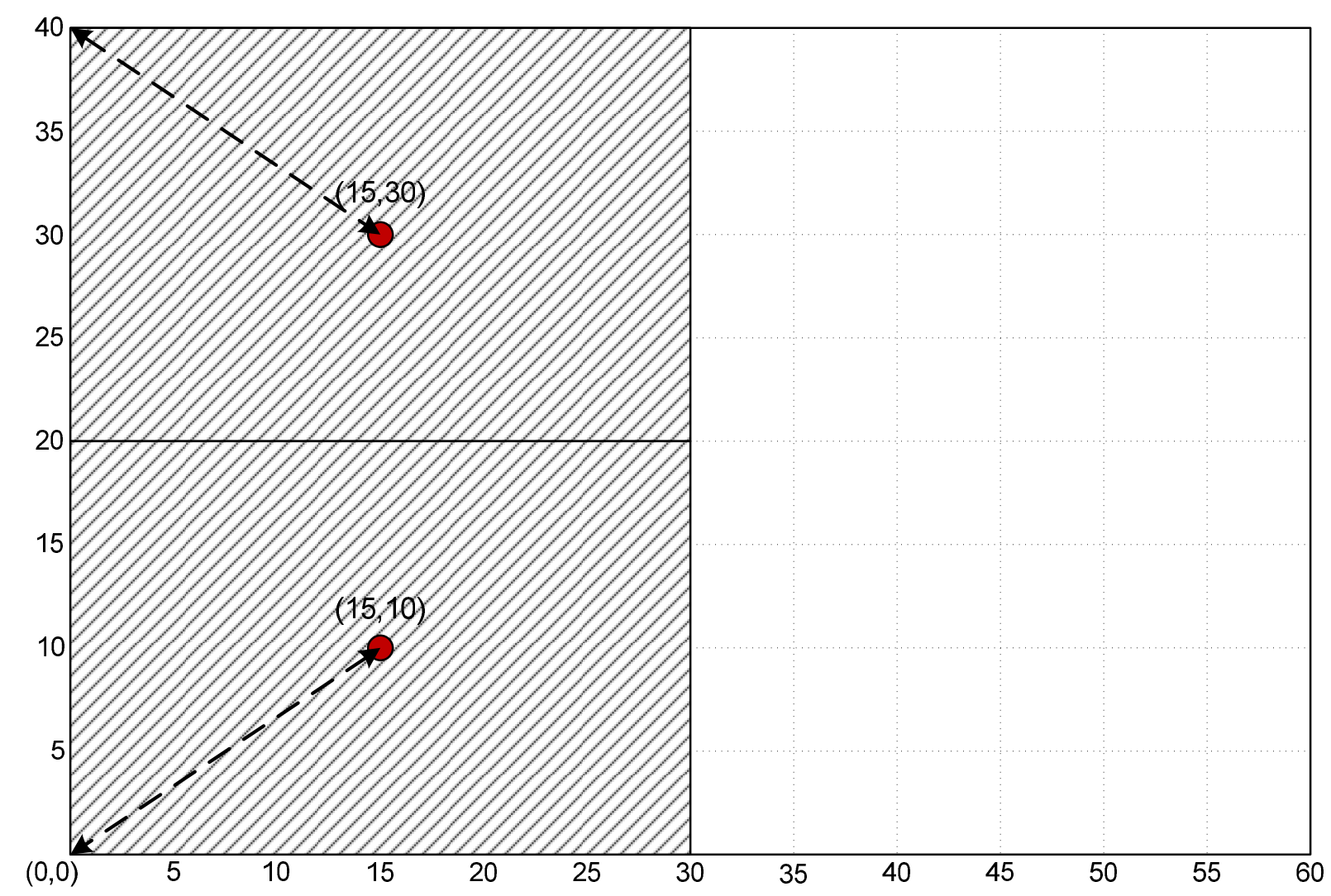

Figure 6.4: CPP Placement for 2 SA's and 2 CPP's and the distance of the worst user.

mentioned case are shown. Although in our problem the CPP's are identical, and the same is for most of the real world scenarios, in this figure, the coverage area of each CPP is considered to be less than that for the case with 4 SA's and 1 CPP. Figure $6.5 \mathrm{~b}$ shows the coverage area of a CPP supporting 4 SA's on a floor. The reason for this difference is to prevent adjacentchannel interference. Although the adjacent CPP's are using non-overlapping channels, if they are too close to each other or using a high output power level, the channels will interfere with each other. In site surveying, in order to determine if the output power level of a base station is high, a protocol analyzer is used with 3 to 4 channels off from the base station and observe the number of packets received by it. In our problem, we assume reducing the output power according to the need of each scenario.

Continuing the scenarios with two CPP's on a single floor, in case we have 3 SA's and 


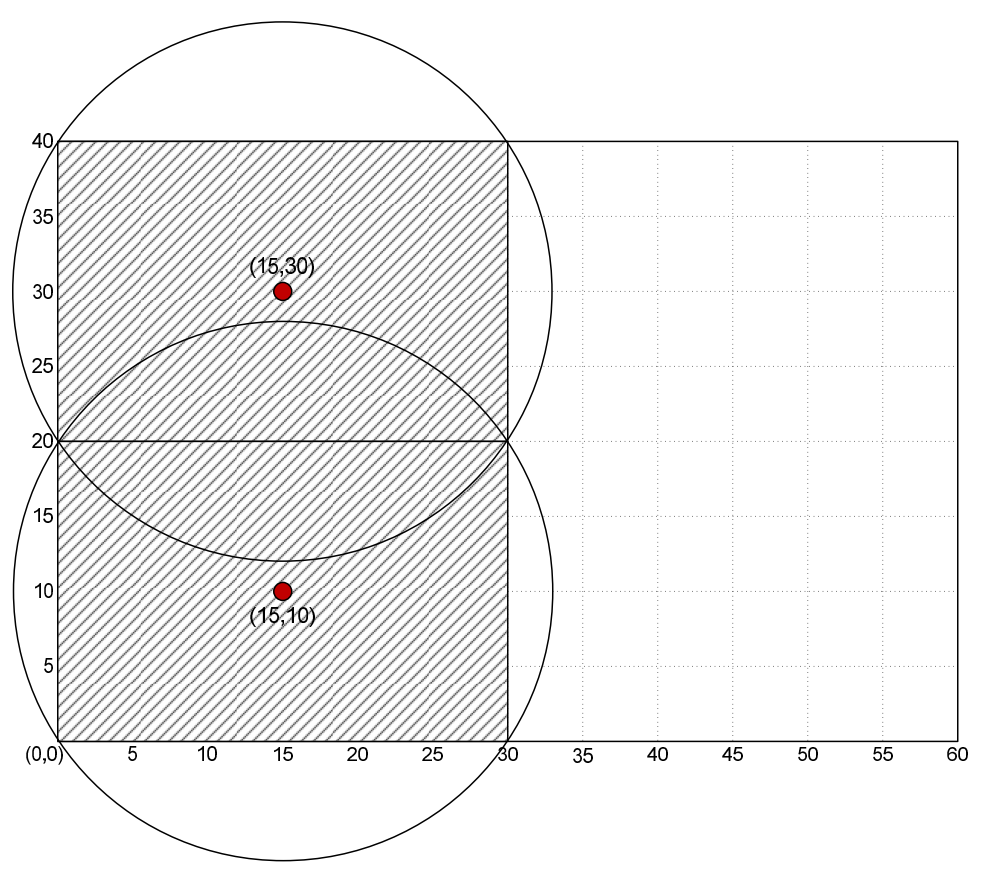

(a) CPP coverage for $2 \mathrm{SA}$ 's and 2 CPP's.

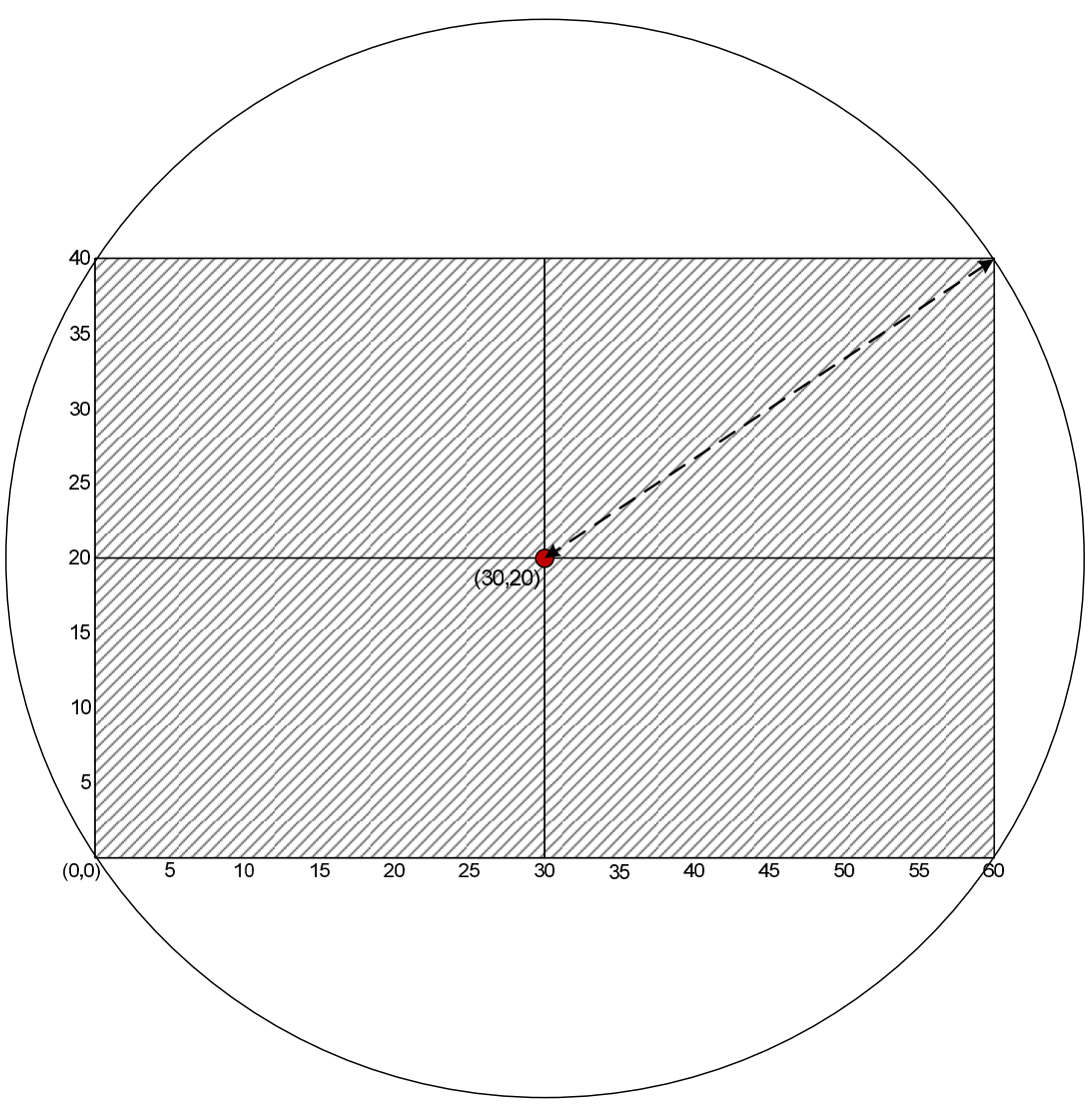

(b) CPP coverage for $4 \mathrm{SA}$ 's and $1 \mathrm{CPP}$.

Figure 6.5: CPP coverage and output power level difference for two different cases. 
2 CPP's, the simulation results return the assignment of one CPP to two of the SA's and assignment of the other CPP to the remaining SA. The CPP positioning from this set of results is shown in Figure 6.6. As can be observed the CPP's are working with the same

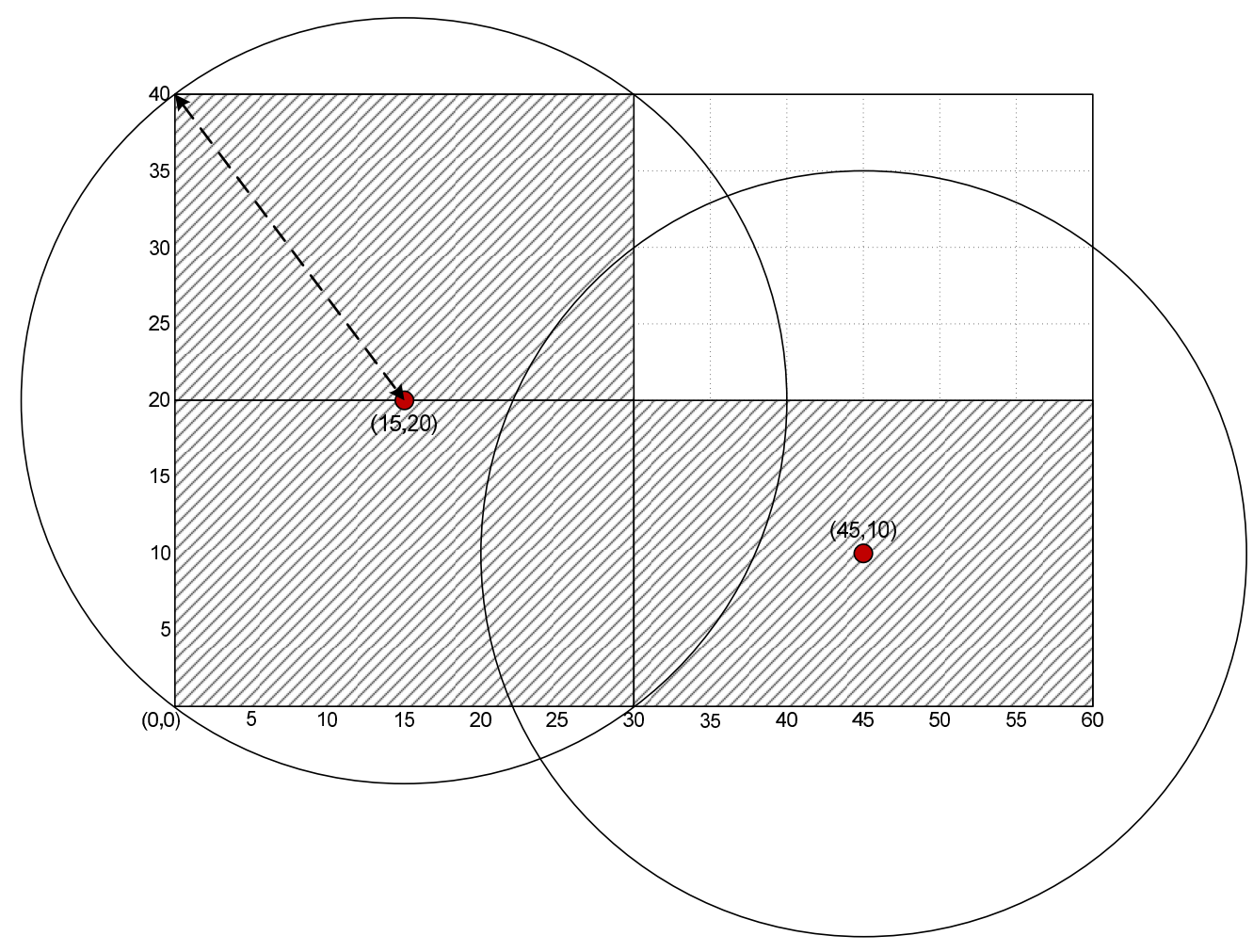

Figure 6.6: CPP positioning and coverage for 3 SA's on a single floor and 2 CPP's.

output power and their coverage is identical. However, according to previous discussion, the output power of the CPP on the right can be reduced in this layout to accommodate adjacent-channel interference avoidance. The transmission power values from $S A 1, S A 2$ and $S A 3$ are $649.51 \mu \mathrm{W}, 648.67 \mu \mathrm{W}$ and $67.94 \mu \mathrm{W}$, respectively.

And finally for the case when we have 4 SA's and 2 CPP's on a single floor, Figure 6.7 demonstrates the CPP placement and assignment of SA's to CPP's. The transmission power of all SA's are within the same range, with the maximum of them being $\lambda=649.88 \mu \mathrm{W}$.

We can now compare the impact of having two CPP's on the transmission power of the SA's to when there is only one CPP. For this, we need to consider the average transmission 


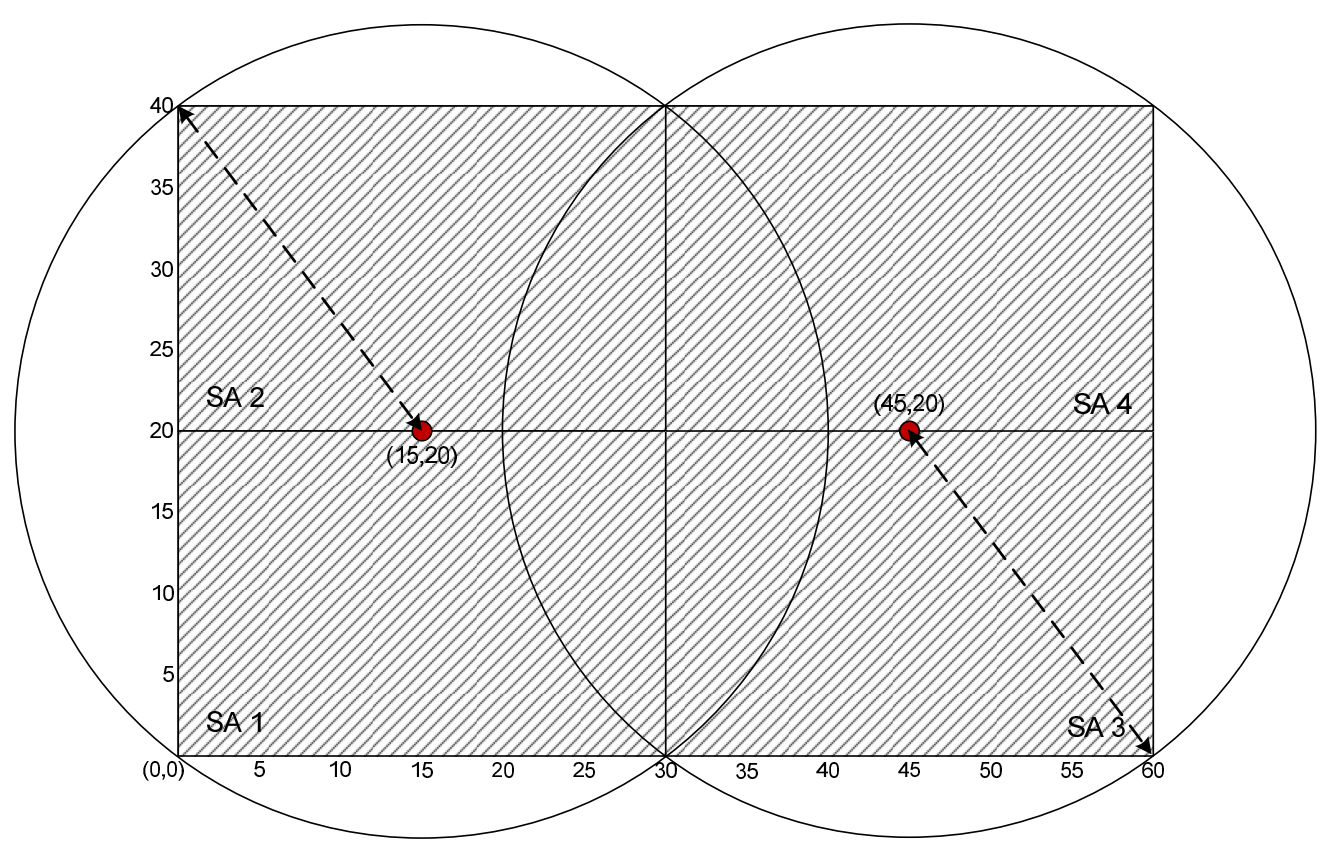

Figure 6.7: CPP positioning and coverage for 4 SA's on a single floor and 2 CPP's.

power of SA's for the case when we have two CPP's and 3 SA's, as this value is different for various SA's: $p_{\text {avg }}^{2,3}=\frac{\left(p_{1}+p_{2}+p_{3}\right)}{3}=\frac{649.51+648.67+67.94}{3}=455.37$

Figure 6.8 shows the reduction in transmission power of SA's when another CPP is added to the same floor.

\subsubsection{More Random SA's}

Now that we have seen and felt results for more intuitive cases, let us consider the setup in Figure 6.9 for when we have two SA's and one CPP. In this figure, the CPP placement is also shown from the results obtained from the simulator. According to these results, the power consumption for the worst user in the left SA is $502 \mu \mathrm{W}$ and that of the worst user in the right $\mathrm{SA}$ is $504 \mu \mathrm{W}$. These values are very close to each other as the distances between the CPP and the furthest points of the SA's are almost identical.

Next, consider the setup and SA-orientation in Figure 6.10. SA 1 has larger width than length. As in other figures of this chapter, the location of the CPP obtained from the 


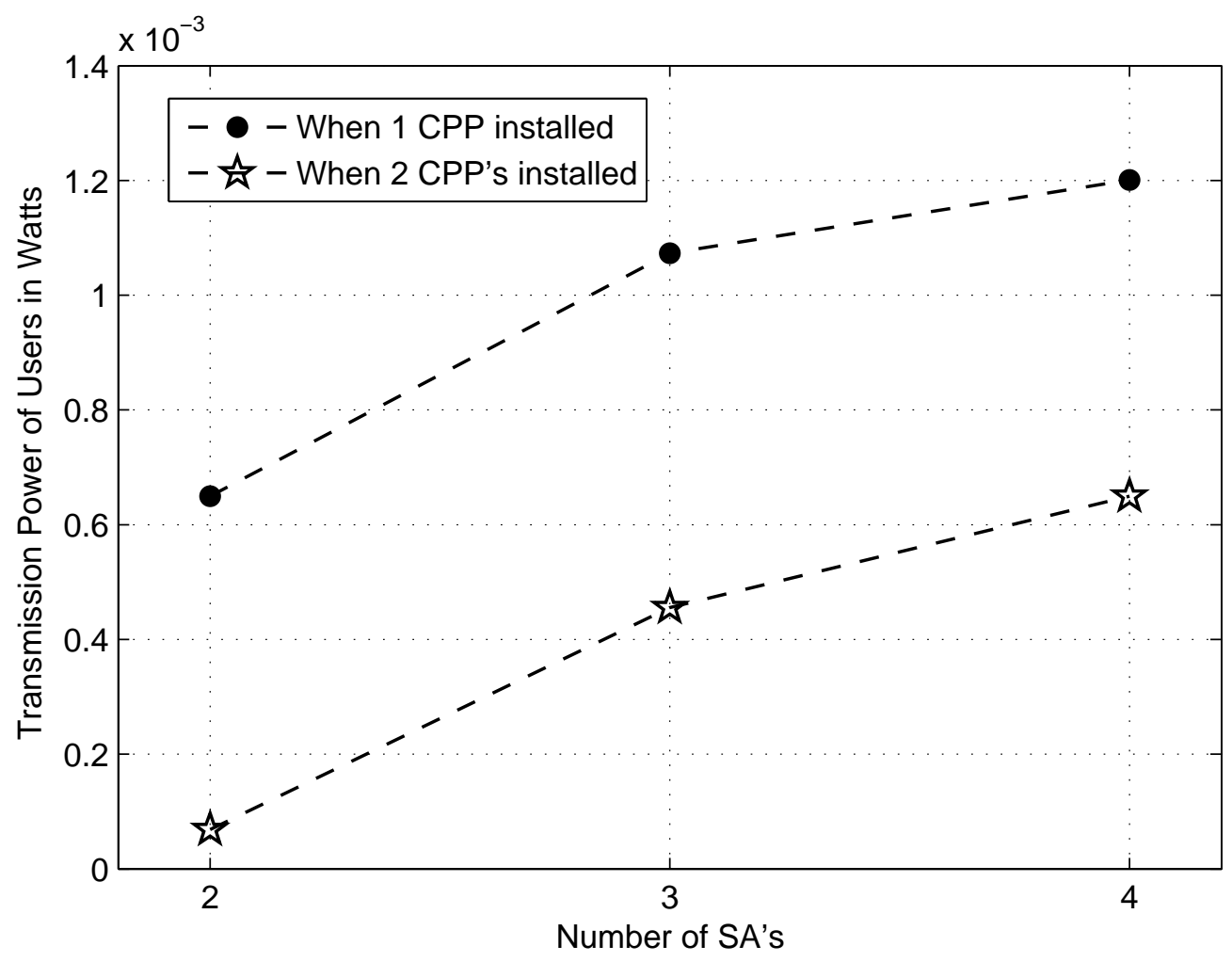

Figure 6.8: Comparison between the average transmission power of SA's in the presence of 2 CPP's (the topology shown in Figure 6.7) and 1 CPP.

simulation results, is shown in this figure as well. The transmission power for the worst user of $S A 1$ is $666 \mu \mathrm{W}$, for $S A 2$ is $612 \mu \mathrm{W}$, and that of $S A 3$ is $667 \mu \mathrm{W}$. Again, the distance between the furthest point of $S A 1$ and the CPP, is identical to that of $S A$ 3. Therefore, the uplink transmission powers from these SA's are identical. On the other hand, this value is lower for $S A 2$.

\subsection{EMI sensitivity}

In Section 6.1, we explained that wireless users are not allowed to enter the guarded area present in our system model. Also we assumed that each SA has only one user. Therefore, if we consider a guarded area as a circle in the middle of an SA, the maximum electric field that can be sensed in the middle of the SA, is due to the user being on the border of the 


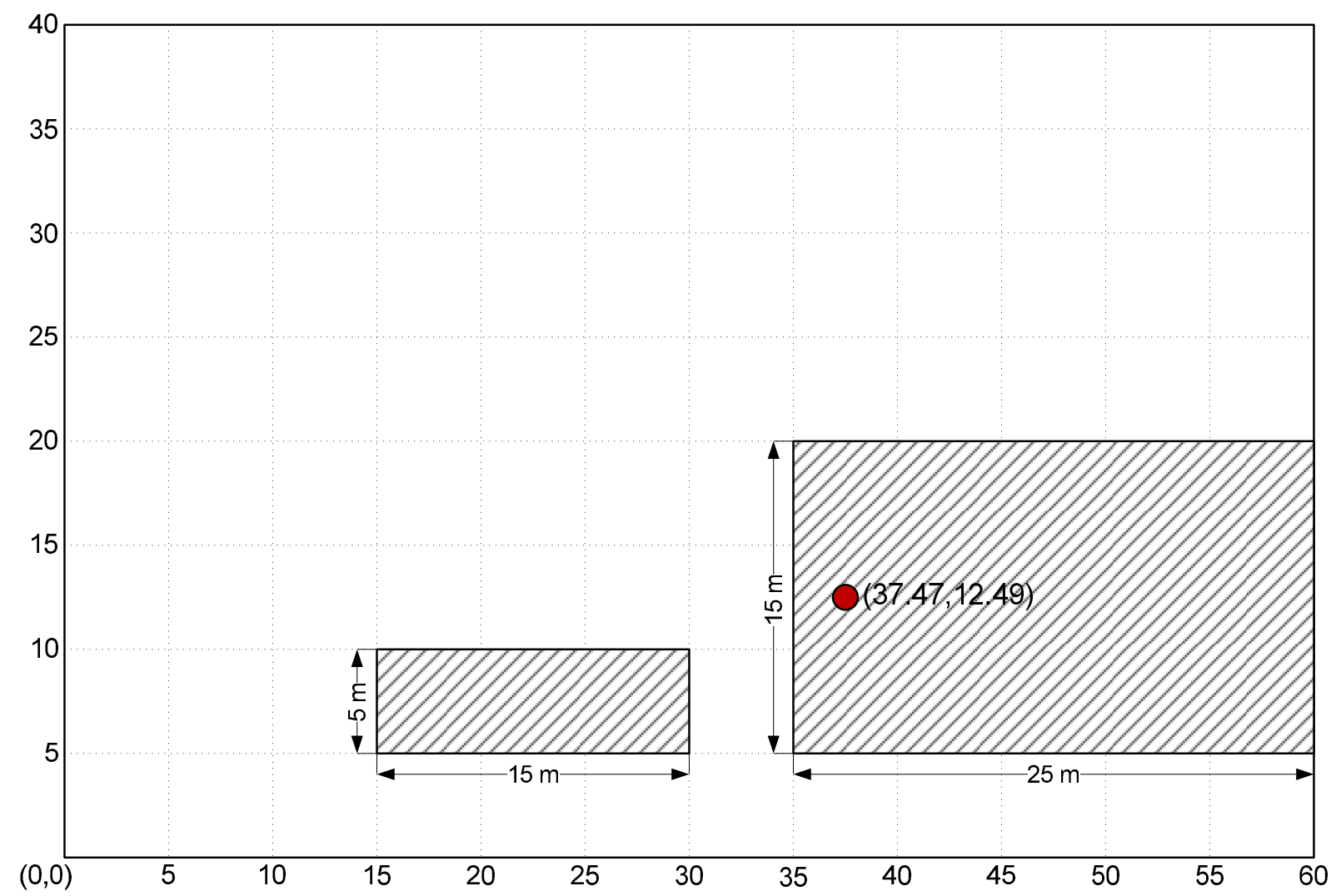

Figure 6.9: Semi-random SA orientation for two SA's and one CPP

guarded area, or the perimeter of the mentioned circle. In this section, we will investigate the effect of the immunity level of medical devices. For this, let us assume the radius of a guarded area to be 0.1 of the length of the SA. In the example of SA's with 30 meters length and 20 meters width, the radius of guarded areas will be 3 meters.

As mentioned in Section 6.1, the immunity level of medical devices was considered to be $I=1$ throughout the simulation. However, in this section we are going to change this value, in order to get a feeling of how the sensitivity of medical devices can affect the transmission power limit for wireless users, and therefore the CPP deployment. Also, $Z$ was considered to be 23 for life-supporting EMI sensitive devices. Let us now assume that we reduce the immunity level 5 times, keeping all other variables untouched. Therefore, $I=0.2$ and from the formula in (5.36), a new upper bound for $p_{n}$ can be calculated: $p_{n} \leq 680 \mu \mathrm{W}$

The value of $680 \mu \mathrm{W}$ for power limit is interesting because it is in between the obtained 
values for $\lambda$ when number of CPP's increase from one to two and there are four SA's. For the case of having one CPP, running the simulation with the new values and logging the results from iterations, showed that there is not an optimal solution for this setup. Every single iteration failed to return a genuine value for the objective function, $\lambda$, while satisfying all the constraints. It is not out of line to mention that the initial guess vector was set for this setup according to the previous experiments.

At this point, it made common sense to increase the number of CPP's in order to reduce the maximum transmission power of wireless users. The optimal results were obtained by increasing the number of CPP's to two, with the value of $651 \mu \mathrm{W}$ returned as the result for objective function, $\lambda$. However, in order to automate the failover, we took advantage of programming skills and intelligence of OPTIToolbox. With the help of the variable maxtime, we limited the time that iterations failed, as failing-iterations sometimes last for a significant

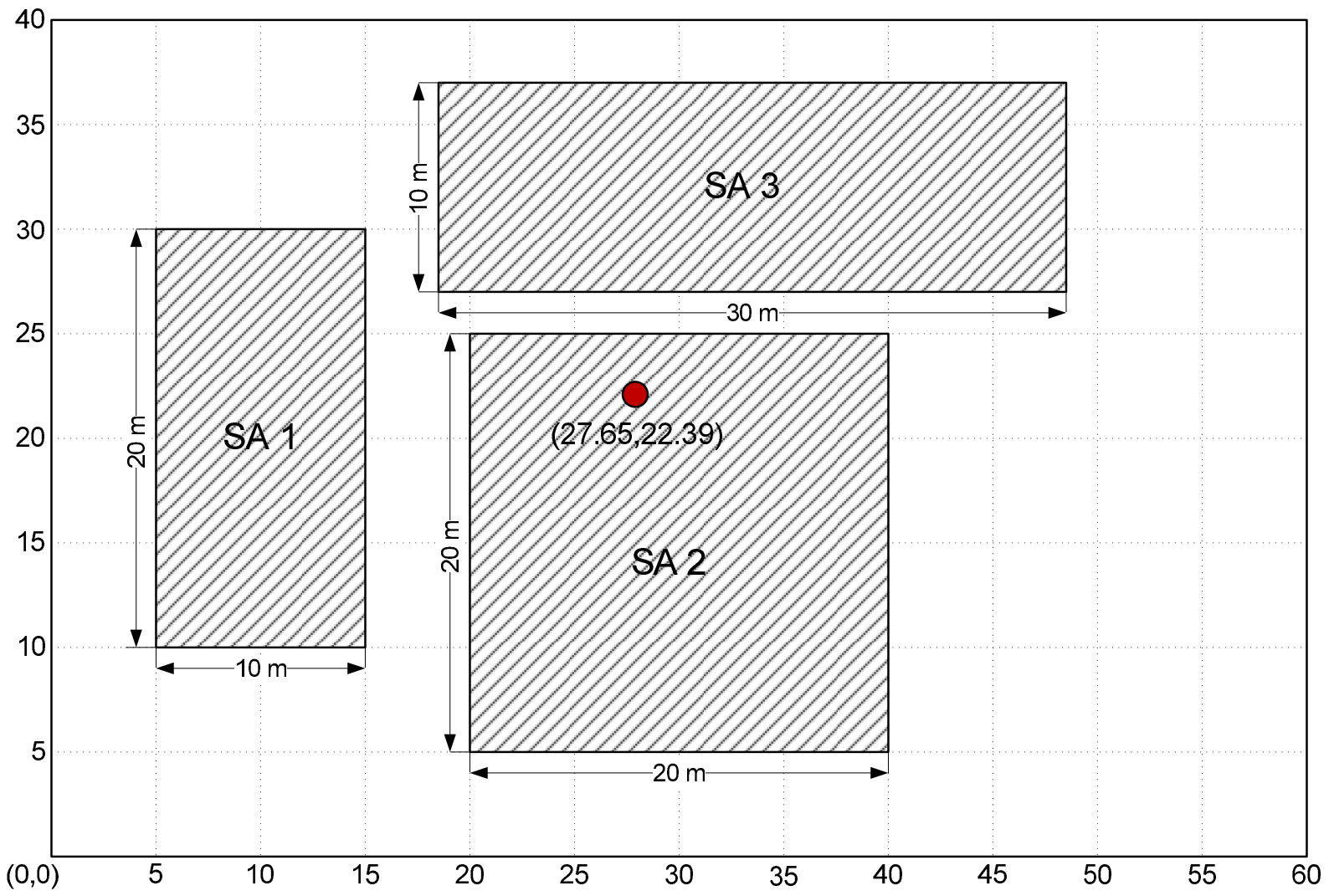

Figure 6.10: Semi-random SA orientation for three SA's and one CPP 
amount of time. Then, by comparing the exitFlag to "0", which is the value for when the time limit is exceeded, we were able to call the main program with increment in the number of CPP's.

This experiment shows that the more sensitive the medical devices are to the EMI, the lower the power limit of wireless transmitters. Therefore, as we only consider the uplink power in our problem, one good resolution would be increasing the number of CPP's which results in reduction of average uplink transmission power.

\subsection{Chapter summary}

The first part of this chapter, was dedicated to calculating and assigning proper values to the variables and constant. The results from running our MATLAB program for a number scenarios, constitute the rest of the chapter. Appropriate change of values and diagram demonstrations are presented in details throughout this chapter. The effect of increasing the number of SA's while the number of CPP's does not change, and vice versa, on the uplink transmission power of wireless users was also investigated. 


\section{Chapter 7}

\section{Conclusion and Future Work}

\subsection{Summary and conclusion}

In this thesis, an approach to solve a multi-objective problem of EMI-aware central processing point $(\mathrm{CPP})$ deployment for wireless sensor networks in hospitals, was presented. The proposed method, formulates a non-convex mixed-integer non-linear programming (MINLP) problem incorporating the following objectives:

- Minimizing the transmission power of wireless transmitters,

- Optimizing the central processing point $(\mathrm{CPP})$ deployment,

- And minimizing the harmful electromagnetic interference (EMI) on sensitive medical devices.

For wireless sensors in a sensor network environment, it is always critical and interesting to minimize transmission power consumption, as the only energy resource for sensor nodes is their limited battery supplies. This is of more importance within clinics, hospital buildings, and field hospitals. On the other hand, minimization of transmission power of wireless nodes is in favor of EMI avoidance on both life-supporting and non-life-supporting medical devices. Therefore, in our formulation, we limited the transmission power according to the 
maximum electric field allowed around the medical devices. On the other hand, we limited the operation of these medical devices to certain areas, called protected areas within service areas (SA's). SA's are region of a hospital floor where the wireless transmitters operate.

Another important objective of this approach is efficient CPP deployment. This will result not only in optimal network coverage in every corner of the SA's, but also in lowering the power consumption of wireless sensor nodes. According to our decision variables, the simulation results also determine the association of SA's to appropriate CPP's.

To solve the formulated MINLP problem, we used a branch-and-bound algorithm suited for our formulation. The coding for simulation done in MATLAB and OPTIToolbox, is used to find the minimum value of objective function in different cases, with a modified branch-and-bound algorithm.

Our numerical results confirm the compliance of transmission power of wireless users with the limitations and constraints imposed by EMI mitigation, CPP deployment scheme, path loss model and CPP association model. It was shown that the number of deployed CPP's increase, as the EMI constraint becomes more stringent and path loss model becomes more complicated. Also, effects of penetration loss through a floor separation was investigated.

\subsection{Future work}

Based on this work, the following are open areas and potential research directions that might be interesting to pursue in the future:

- In our system model and in most of our simulation setups, we only investigated wireless sensor network on a single-floor hospital building. Similar network in a multi-storey hospital would be a good direction to consider for future work.

- Integrating tele-health services and collecting data from CPP's by a central controller for submitting the aggregated data over the Internet is one of interesting topics that is under-explored. 
- We assumed only two kinds of users, sensor nodes with wireless transmitter and protected medical devices. Transmitters can be further divided into two categories: primary users and secondary users. Primary users would consist of medical sensors and devices that have to transmit wireless data. This data is usually of high importance and have to be real-time. Thus, primary users will have prioritized access to the channel, whereas secondary users may take advantage from opportunistic channel access. Secondary users would consist of data aggregators, laptop users, etc. Other than channel access problem, in this context, the interference mitigation can be remodeled as the interference from secondary users to primary and protected users, and the interference from primary users to protected users.

- For numerical analysis, we assumed having only one user within each SA with the largest uplink transmission power. This work can be extended by considering multiple users and the accumulated uplink power of them.

- We have deployed a standard branch-and-bound algorithm. As a future research, the effect of different algorithms on results can be investigated. 


\section{Bibliography}

[1] A. Musa, Y. Yusuf, and M. Meckel, "A hospital resource and patient management system based on real-time data capture and intelligent decision making," in Systems and Informatics (ICSAI), 2012 International Conference on, pp. 776-779, 2012.

[2] E. Sibarani, "Simulating an integration systems: Hospital information system, radiology information system and picture archiving and communication system," in Uncertainty Reasoning and Knowledge Engineering (URKE), 2012 2nd International Conference on, pp. 62-66, 2012.

[3] P. Gonzalez-Brevis, J. Gondzio, Y. Fan, H. Poor, J. Thompson, I. Krikidis, and P.-J. Chung, "Base station location optimization for minimal energy consumption in wireless networks," in Vehicular Technology Conference (VTC Spring), 2011 IEEE 73rd, pp. 15,2011 .

[4] A. Torrado-Carvajal, M. Rodriguez-Sanchez, A. Rodriguez-Moreno, S. Borromeo, C. Garro-Gomez, J. Hernandez-Tamames, and M. Luaces, "Changing communications within hospital and home health care," in Engineering in Medicine and Biology Society (EMBC), 2012 Annual International Conference of the IEEE, pp. 6074-6077, 2012.

[5] J. Liu, T. Kou, Q. Chen, and H. Sherali, "Femtocell base station deployment in commercial buildings: A global optimization approach," Selected Areas in Communications, IEEE Journal on, vol. 30, no. 3, pp. 652-663, 2012. 
[6] P. Phunchongharn, E. Hossain, and S. Camorlinga, "Electromagnetic interference-aware transmission scheduling and power control for dynamic wireless access in hospital environments," Information Technology in Biomedicine, IEEE Transactions on, vol. 15, no. 6, pp. 890-899, 2011.

[7] S. Torkestani, S. Sahuguede, A. Julien-Vergonjanne, and J. Cances, "Indoor optical wireless system dedicated to healthcare application in a hospital," Communications, IET, vol. 6, no. 5, pp. 541-547, 2012.

[8] R. De Francisco, "Indoor channel measurements and models at 2.4 ghz in a hospital," in Global Telecommunications Conference (GLOBECOM 2010), 2010 IEEE, pp. 1-6, 2010.

[9] A. Kumar, "Location of mobile user in an indoor wireless system operating in 2 ghz frequency band," in Wireless Communication, Vehicular Technology, Information Theory and Aerospace Electronic Systems Technology, 2009. Wireless VITAE 2009. 1st International Conference on, pp. 294-297, 2009.

[10] D. DePoy and T. Bose, "Predictive behavior classification for cognitive radio: Introduction and preliminary results," in Cognitive Radio Oriented Wireless Networks and Communications (CROWNCOM), 2012 rth International ICST Conference on, pp. 280-284, 2012.

[11] S. Wang, H. Liu, L. Xie, and W. Hu, "Cognitive radio simulation environment realization based on autonomic communication," in Communication Software and Networks (ICCSN), 2011 IEEE 3rd International Conference on, pp. 402-407, 2011.

[12] Y. Tawk, J. Costantine, E. Al-Zuraiqi, and C. Christodoulou, "Cognitive radio antennas that learn and adapt using neural networks," in Radio Science Meeting (USNC-URSI NRSM), 2013 US National Committee of URSI National, pp. 1-1, 2013. 
[13] N. Devroye, M. Vu, and V. Tarokh, "Cognitive radio networks," Signal Processing Magazine, IEEE, vol. 25, no. 6, pp. 12-23, 2008.

[14] X.-S. Yang, Introduction to Mathematical Optimization: From Linear Programming to Metaheuristics. Cambridge International Science Publishing, 2008.

[15] C. A. Floudas, Nonlinear and Mixed-Integer Optimization: Fundamentals and Applications. Nonlinear and Mixed-integer Optimization: Fundamentals and Applications, Oxford University Press, USA, 1995.

[16] J. Nocedal and S. J. Wright, Numerical Optimization. New York: Springer, 2nd ed., 2006.

[17] Y. He, W. Zhu, and L. Guan, "Optimal resource allocation for pervasive health monitoring systems with body sensor networks," Mobile Computing, IEEE Transactions on, vol. 10 , pp. $1558-1575$, nov. 2011.

[18] J. C. Healy, "Implementing e-health in developing countries, guidance and principles," 2008.

[19] R. Istepanian, S. Laxminarayan, and C. Pattichis, M-Health: Emerging Mobile Health Systems. Topics in Biomedical Engineering. International Book Series, Springer, 2010.

[20] V. W. Consulting, "mhealth for development: The opportunity of mobile technology for healthcare in the developing world," Clinics in Dermatology, 2009.

[21] H. Alemdar and C. Ersoy, "Wireless sensor networks for healthcare: A survey," Computer Networks, vol. 54, no. 15, pp. $2688-2710,2010$.

[22] J. Ko, C. Lu, M. Srivastava, J. Stankovic, A. Terzis, and M. Welsh, "Wireless sensor networks for healthcare," Proceedings of the IEEE, vol. 98, pp. 1947 -1960, nov. 2010.

[23] "Newham wsd trial - improving quality of life in newham," 2012. 
[24] A. Steventon, M. Bardsley, J. Billings, J. Dixon, H. Doll, S. Hirani, M. Cartwright, L. Rixon, M. Knapp, C. Henderson, A. Rogers, R. Fitzpatrick, J. Hendy, and S. Newman, "Effect of telehealth on use of secondary care and mortality: findings from the whole system demonstrator cluster randomised trial," BMJ, vol. 344, 62012.

[25] A. Zvikhachevskaya and G. Markarian, Advances in Telemedicine: Technologies, Enabling Factors and Scenarios. Intech.

[26] O. Gama, P. Carvalho, J. A. Afonso, and P. M. Mendes, "Quality of service support in wireless sensor networks for emergency healthcare services," in Engineering in Medicine and Biology Society, 2008. EMBS 2008. 30th Annual International Conference of the IEEE, pp. $1296-1299$, aug. 2008.

[27] E. Park and H. S. Nam, "A service-oriented medical framework for fast and adaptive information delivery in mobile environment," Information Technology in Biomedicine, IEEE Transactions on, vol. 13, pp. 1049 -1056, nov. 2009.

[28] "Philips heartstart mrx als monitor," 2006.

[29] I. Lamprinos, A. Prentza, E. Sakka, and D. Koutsouris, "Energy-efficient mac protocol for patient personal area networks," in Engineering in Medicine and Biology Society, 2005. IEEE-EMBS 2005. 27th Annual International Conference of the, pp. 3799 -3802, jan. 2005.

[30] H. Fariborzi and M. Moghavvemi, "Architecture of a wireless sensor network for vital signs transmission in hospital setting," in Convergence Information Technology, $200 \%$. International Conference on, pp. 745 -749, nov. 2007.

[31] W.-Y. Chung, C.-L. Yau, K.-S. Shin, and R. Myllyla, "A cell phone based health monitoring system with self analysis processor using wireless sensor network technology," in Engineering in Medicine and Biology Society, 200\%. EMBS 200\%. 29th Annual International Conference of the IEEE, pp. 3705 -3708, aug. 2007. 
[32] U. Varshney, "Pervasive healthcare and wireless health monitoring," Springer Science + Business Media, LLC, vol. 12, pp. 113 -127, jul. 2007.

[33] M. El Khaddar, H. Harroud, M. Boulmalf, M. Elkoutbi, and A. Habbani, "Emerging wireless technologies in e-health trends, challenges, and framework design issues," in Multimedia Computing and Systems (ICMCS), 2012 International Conference on, pp. $440-445$, may 2012 .

[34] Y. He, W. Zhu, and L. Guan, "Optimal resource allocation to provide qos guarantee in pervasive health monitoring systems," in Multimedia and Expo (ICME), 2011 IEEE International Conference on, pp. 1 -6, july 2011.

[35] D. Vouyioukas, I. Maglogiannis, and D. Komnakos, "Emergency m-health services through high-speed $3 \mathrm{~g}$ systems: Simulation and performance evaluation," SIMULATION, pp. $329-345$, Apr. 2007.

[36] J. Stankovic, Q. Cao, T. Doan, L. Fang, Z. He, R. Kiran, S. Lin, S. Son, R. Stoleru, and A. Wood, "Wireless sensor networks for in-home healthcare: Potential and challenges." High Confidence Medical Device Software and Systems (HCMDSS) Workshop, 2005.

[37] Y. Zhang and H. Xiao, "Bluetooth-based sensor networks for remotely monitoring the physiological signals of a patient," Information Technology in Biomedicine, IEEE Transactions on, vol. 13, pp. 1040 -1048, nov. 2009.

[38] L. Traver, C. Tarin, and N. Cardona, "Bandwidth resource management for neural signal telemetry," Information Technology in Biomedicine, IEEE Transactions on, vol. 13, pp. $1083-1084$, nov. 2009.

[39] B. Shrestha, E. Hossain, and S. Camorlinga, "Ieee 802.15.4 mac with gts transmission for heterogeneous devices with application to wheelchair body-area sensor networks," Information Technology in Biomedicine, IEEE Transactions on, vol. 15, pp. $767-777$, sept. 2011. 
[40] M. Fengou, G. Mantas, D. Lymberopoulos, N. Komninos, S. Fengos, and N. Lazarou, "A new framework architecture for next generation e-health services," Information Technology in Biomedicine, IEEE Transactions on, vol. PP, no. 99, p. 1, 2012.

[41] L. Skorin-Kapov and M. Matijasevic, "Analysis of qos requirements for e-health services and mapping to evolved packet system qos classes," International Journal of Telemedicine and Applications, vol. 2010, pp. 1-18, jul. 2010.

[42] B. Lo and G.-Z. Yang, "Body sensor networks - research challenges and opportunities," in Antennas and Propagation for Body-Centric Wireless Communications, 2007 IET Seminar on, pp. $26-32$, april 2007.

[43] K. M. Siassiakos and A. A. Lazakidou, Handbook of Research on Distributed Medical Informatics and E-Health. IGI Global.

[44] A. Umamageswari and G. Suresh, "Security in medical image communication with arnold's cat map method and reversible watermarking," in Circuits, Power and Computing Technologies (ICCPCT), 2013 International Conference on, pp. 1116-1121, 2013.

[45] L. Qiao and P. Koutsakis, "Adaptive bandwidth reservation and scheduling for efficient wireless telemedicine traffic transmission," Vehicular Technology, IEEE Transactions on, vol. 60, pp. $632-643$, feb. 2011.

[46] A. Bhargava, M. Farrukh Khan, and A. Ghafoor, "Qos management in multimedia networking for telemedicine applications," in Software Technologies for Future Embedded Systems, 2003. IEEE Workshop on, pp. 39 - 42, may 2003.

[47] I. Martinez, J. Garcia, and E. Viruete, "Resources variability in m-health services: An adaptive method for qos control," in Consumer Communications and Networking Conference, 2008. CCNC 2008. 5th IEEE, pp. 829 -833, jan. 2008. 
[48] G. Cova, X. Huagang, G. Qiang, E. Guerrero, R. Ricardo, and J. Estevez, "A perspective of state-of-the-art wireless technologies for e-health applications," in IT in Medicine Education, 2009. ITIME '09. IEEE International Symposium on, vol. 1, pp. 76 -81, aug. 2009.

[49] G. Markarian, L. Mihaylova, D. Tsitserov, and A. Zvikhachevskaya, "Video distribution techniques over wimax networks for m-health applications," Information Technology in Biomedicine, IEEE Transactions on, vol. 16, pp. 24 -30, jan. 2012.

[50] K. Kawamura, Y. Kobayashi, and M. Fujie, "Development of real-time simulation for workload quantization in robotic tele-surgery," in Robotics and Biomimetics, 2006. ROBIO '06. IEEE International Conference on, pp. 1420 -1425, dec. 2006.

[51] G. Laleci and A. Dogac, "A semantically enriched clinical guideline model enabling deployment in heterogeneous healthcare environments," Information Technology in Biomedicine, IEEE Transactions on, vol. 13, pp. 263 -273, march 2009.

[52] M. Barua, M. Alam, X. Liang, and X. Shen, "Secure and quality of service assurance scheduling scheme for wban with application to ehealth," in Wireless Communications and Networking Conference (WCNC), 2011 IEEE, pp. 1102 -1106, march 2011.

[53] T. Gao, T. Massey, L. Selavo, D. Crawford, B.-R. Chen, K. Lorincz, V. Shnayder, L. Hauenstein, F. Dabiri, J. Jeng, A. Chanmugam, D. White, M. Sarrafzadeh, and M. Welsh, "The advanced health and disaster aid network: A light-weight wireless medical system for triage," Biomedical Circuits and Systems, IEEE Transactions on, vol. 1, pp. $203-216$, sept. 2007.

[54] H. Ng, M. Sim, C. Tan, and C. Wong, "Wireless technologies for telemedicine," BT Technology Journal, vol. 24, pp. 130-137, 2006. 
[55] A. Zvikhachevskaya, G. Markarian, and L. Mihaylova, "Quality of service consideration for the wireless telemedicine and e-health services," in Wireless Communications and Networking Conference, 2009. WCNC 2009. IEEE, pp. 1-6, april 2009.

[56] U. Mitra, B. Emken, S. Lee, M. Li, V. Rozgic, G. Thatte, H. Vathsangam, D. Zois, M. Annavaram, S. Narayanan, M. Levorato, D. Spruijt-Metz, and G. Sukhatme, "Knowme: a case study in wireless body area sensor network design," Communications Magazine, IEEE, vol. 50, pp. 116 -125, may 2012.

[57] S. Weber, J. Andrews, X. Yang, and G. de Veciana, "Transmission capacity of wireless ad hoc networks with successive interference cancellation," Information Theory, IEEE Transactions on, vol. 53, pp. 2799 -2814, aug. 2007.

[58] M. A. Khalid and K. K. Chai, "Performance comparison of ad hoc routing protocols in e-health applications," Computer Science Journal, vol. 2, April 2012.

[59] J. Andrews, "Interference cancellation for cellular systems: a contemporary overview," Wireless Communications, IEEE, vol. 12, pp. 19 - 29, april 2005.

[60] O. Omeni, O. Eljamaly, and A. Burdett, "Energy efficient medium access protocol for wireless medical body area sensor networks," in Medical Devices and Biosensors, $200 \%$. ISSS-MDBS 200\%. 4th IEEE/EMBS International Summer School and Symposium on, pp. $29-32$, aug. 2007.

[61] Y. Wang and K. Chang, "Novel radio resource management schemes for best-effort service in ofdma systems," in Communications (MICC), 2009 IEEE 9th Malaysia International Conference on, pp. 580-585, 2009.

[62] X. Yang, T. Lehman, C. Tracy, J. Sobieski, S. Gong, P. Torab, and B. Jabbari, "Policybased resource management and service provisioning in gmpls networks," in INFOCOM 2006. 25th IEEE International Conference on Computer Communications. Proceedings, pp. 1-12, 2006. 
[63] T. Zahir, K. Arshad, A. Nakata, and K. Moessner, "Interference management in femtocells," Communications Surveys Tutorials, IEEE, vol. PP, no. 99, pp. 1 -19, 2012.

[64] M. Yavuz, F. Meshkati, S. Nanda, A. Pokhariyal, N. Johnson, B. Raghothaman, and A. Richardson, "Interference management and performance analysis of umts/hspa+ femtocells," Communications Magazine, IEEE, vol. 47, pp. 102 -109, september 2009.

[65] N. Saquib, E. Hossain, L. B. Le, and D. I. Kim, "Interference management in ofdma femtocell networks: issues and approaches," Wireless Communications, IEEE, vol. 19, pp. $86-95$, june 2012 .

[66] Y. Li, H. Zhang, and T. Asami, "On the cooperation between cognitive radio users and femtocell networks for cooperative spectrum sensing and self-organization," in Wireless Communications and Networking Conference (WCNC), 2013 IEEE, pp. 446-451, 2013.

[67] J. Miranda, R. Abrishambaf, T. Gomes, P. Goncalves, J. Cabral, A. Tavares, and J. Monteiro, "Path loss exponent analysis in wireless sensor networks: Experimental evaluation," in Industrial Informatics (INDIN), 2013 11th IEEE International Conference on, pp. 54-58, 2013.

[68] T. Rappaport, Wireless Communications: Principles and Practice. Upper Saddle River, NJ, USA: Prentice Hall PTR, 2nd ed., 2002.

[69] P. Bonami, L. T. Biegler, A. R. Conn, G. Cornujols, I. E. Grossmann, C. D. Laird, J. Lee, A. Lodi, F. Margot, N. Sawaya, and A. Wchter, "An algorithmic framework for convex mixed integer nonlinear programs," Discrete Optimization, vol. 5, no. 2, pp. 186 $-204,2008$.

[70] E. Kalvelagen, "Some minlp solution algorithms," 2003. 